Canadian University Music Review

Revue de musique des universités canadiennes

\title{
Des neumes à la portée
}

Élaboration et organisation rationnelles de la discontinuité

musicale et de sa représentation graphique, de la formule mélodique à l'échelle monocordale

\section{Marie-Elisabeth Duchez}

\section{Numéro 4, 1983}

URI : https://id.erudit.org/iderudit/1013897ar

DOI : https://doi.org/10.7202/1013897ar

Aller au sommaire du numéro

\section{Éditeur(s)}

Canadian University Music Society / Société de musique des universités canadiennes

\section{ISSN}

0710-0353 (imprimé)

2291-2436 (numérique)

Découvrir la revue

Citer cet article

Duchez, M.-E. (1983). Des neumes à la portée : élaboration et organisation rationnelles de la discontinuité musicale et de sa représentation graphique, de la formule mélodique à l'échelle monocordale. Canadian University Music Review / Revue de musique des universités canadiennes, (4), 22-65. https://doi.org/10.7202/1013897ar

(c) Canadian University Music Society / Société de musique des universités canadiennes, 1983
Ce document est protégé par la loi sur le droit d'auteur. L'utilisation des services d'Érudit (y compris la reproduction) est assujettie à sa politique d'utilisation que vous pouvez consulter en ligne.

https://apropos.erudit.org/fr/usagers/politique-dutilisation/ 


\section{DES NEUMES À LA PORTÉE Elaboration et organisation rationnelles de la discontinuité musicale et de sa représentation graphique, de la formule mélodique à l'échelle monocordale}

\section{Marie-Elisabeth Duchez}

\section{MÉMOIRE ET NOTATION : LE RECOURS Ả L'ÉCRITURE MUSICALE ${ }^{1}$}

1) Le problème de la mémorisation du chant liturgique au IXème siècle

Les principes généraux de la notation diastématique classique de notre musique traditionnelle, tant instrumentale que vocale, ont été élaborés du IXème au XIème siècle sous la pression des nécessités pratiques de la transmission orale de la musique: ils sont le résultat de recherches de solutions concrètes à la mémorisation du chant de l'office chrétien, puis de son ornementation polyphonique. C'est donc le problème quotidien de la mnémotechnie d'une musique exclusivement vocale, monodique au départ, et liée phénoménalement et structuralement au langage sacré qu'elle a pour rôle de soutenir et de magnifier, qui va déterminer pour un millénaire les bases de notre représentation graphique de la musique ; et, qui va, par la même exigence, déterminer les fondements rationnels de notre représentation théorique de la musique. En effet, tout, ou presque tout, dans la théorie musicale construite pendant la période du Haut Moyen-Âge, est déterminé par le passage nécessaire de la tradition orale à la musique écrite ; et l'étude de cette construction pourrait être organisée autour de ce pivot épistémologique qui est, avec la polyphonie, la caractéristique de la musique occidentale.

La transmission orale de la musique, collective, officielle rigoureusement et religieusement fidèle, n'est possible que si 
l'émetteur et le récepteur sont en présence l'un de l'autre, donc coïncident dans l'espace et dans le temps; d'autre part, elle dépend des capacités de stockage de la mémoire. Et il ne s'agit pas de la mémoire musicale à court terme, élément même de la musique, qui agit nécessairement et sans relâche, dans un balancement rétrospectif et prospectif, pour donner forme et signification à toute émission et perception de la musique ; mais de la mémoire musicale à long terme (qui a d'ailleurs fait employer la musique elle-même comme moyen de mémorisation), ${ }^{2}$ dans laquelle les hommes du Haut Moyen-Âge ont, à la suite de Saint Augustin, vu une condition d'existence de la musique, dont celleci tient son nom, sa permanence, et par là sa faiblesse.

Insistant sur le caractère transitoire et fugitif du son que retient la mémoire, Augustin rappelle, dans le De ordine, le mythe poétique qui fait des Muses, donc de la Musique tenant d'elles son nom, les filles du Jupiter et de la Mémoire. ${ }^{3} \mathrm{Il}$ contestera, dans le De doctrina christiana (1949 : L.II, XVII, 27), ce mythe de l'origine de la musique comme une erreur des gentils (1948 : 282-83), mais ses formules seront rabâchées pendant des siècles, d'après Cassiodore (1937:140) et surtout d'après Isidore de Séville. Isidore, reprenant le texte d'Augustin, ajoute que « si les sons ne sont pas retenus par l'homme dans sa mémoire, ils se perdent parce qu'ils ne peuvent pas être écrits ${ }^{4}{ }^{4}$

Je cite ce texte, bien que très connu, car il est extrêmement important historiquement, nous indiquant l'impossibilité d'une graphie musicale au VIIème siècle. Notons que si l'absence d'écriture musicale au VIIème siècle, parfois contestée (Don G. Sunyol, W. Gurlitt, Constantin Floros, etc.), est généralement reconnue par la plupart des musicologues (Peter Wagner, Johannes Wolf, J. Chailley, A. Machabey, H. Huschen, etc.), l'impossibilité d'écrire les sons de la voix non parlée (voix chantée, cri d'animaux) était affirmée déjà par les auteurs du IVème siècle qu'Isidore a compilés : Pères de l'Eglise comme Lactance, ou Grammairiens comme Diomède, Cledonius, Marius Victorinus, Priscien. Dans le courant du IXème siècle, lorsque l'écriture neumatique s'est répandue, la phrase affirmant l'impossibilité d'une graphie musicale disparait des citations et des adaptations : on la trouve encore chez Rhaban Maur (s.d. : 495), on ne la trouve plus chez Aurélien de Réomé qui écrit seulement que la musique n'est pas conservée si elle n'est pas gravée dans la mémoire. ${ }^{5}$

La mémorisation d'un répertoire oral exige un effort vital 
bien plus grand que la lecture d'une répertoire écrit. Et dans l'exécution du chant grégorien - exécution qui consistait à assembler sur le texte liturgique écrit, par les procédés pratiques d'ajustage de la modalité empirique, des formules musicales traditionnelles en nombre assez restreint et bien fixées par la mémoire, soit à chanter par cour des pièces entières où se retrouvaient ces mêmes formules - la mémorisation musicale était moins difficile pour le psalmiste qui chantait les psaumes avec leurs Antiennes et leurs Répons dans un cadre simple préalablement mémorisé, que pour le spécialiste qui chantait les Traits et les Graduels d'une structure formulaire plus élaborée. N'intervenait alors qu'une mémoire empirique, ${ }^{6}$ immédiate, intuitive et instinctive, mémoire purement sonore, "mémoire de nature organique ${ }^{7}$ fondée sur l'imitation, " mémoire des oiseaux et des chanteurs ignorants " que Saint Augustin (De ordine, L.II, XIX, 49) oppose à la mémoire rationnelle du savant et du Musicus qui est une mémoire conceptuelle, raisonnée, discursive, médiate et exprimable par l'écriture (1948:448-49). La pédagogie viva voce du chant liturgique consistait en de très nombreuses répétitions de l'exécution du maître et impliquait un très long apprentissage, plus de dix ans ${ }^{8}$; et réapprendre d'un chant oublié ou apprendre un nouveau chant exigeait l'intervention d'un chantre voisin. ${ }^{9}$ Cet enseignement cum voce magistri était encore le seul au IXème siècle, et il se prolongea jusqu'au XIème siècle, jusqu'à l'établissement de la notation diastématique sur portée, dont le promoteur Gui d'Arezzo affirme que, jusqu'à l'introduction de ses innovations, les chanteurs n'auraient pas pu apprendre sine voce magistri "même s'ils s'exerçaient à l'étude du chant pendant cent ans » $(1955: 86$ et $1975: 61)$. A cette initiation par imitation, le maître devait sans doute, afin d'aider ses élèves, ajouter des indications orales qui, jointes aux gestes de la chironomie (ad instar manu) (cf. Aurélien de Réomé 1975: 123), tentaient de donner une description du mouvement de la voix (motus vocis ou vocum), pour en faciliter la reproduction. Cette description naïve était faite dans le langage ordinaire (le latin, langue de la liturgie et du savoir), sans terminologie spéciale adéquate, à l'aide des métaphores attachées à la perception (métaphores coenesthésiques et kinesthésiques). ${ }^{10}$ Cette tentative de représentation sommaire de la conduite de la voix (ductio, productio), que j'ai appelée verbo-gestuelle et dont nous trouvons des témoignages écrits depuis le VIIème siècle (avec Isidore de Séville), marque les premiers essais d'objectivation de 
la musique. Mais la transmission d'une répertoire musical que le développement de la liturgie avait considérablement accru, dépassa bientôt les possibilités de la tradition orale qui avait suffi jusque là. Et c'est ce souci de pédagogie musicale pratique qui est à l'origine des recherches de moyens mnémotechniques empiriques, puis, face à l'échec de ces premières stratégies de la mémoire, à l'origine d'une réflexion décisive fournissant des moyens mnémotechniques rationnels.

Le besoin d'amplifier la mémorisation fut donc, durant ces mêmes VIIème-IXème siècles, le point de départ d'une première pédagogie empirique, née de la pratique musicale. Pour guider plus efficacement la conduite du chant, toujours lié essentiellement au langage et pratiquement indistinct de la prononciation (pronuntiatio) du texte, on eut recours aux notions, aux catégories et aux méthodes (définition, différentiation, classification) de la grammaire, qui régissait le texte verbal et qui, dominant alors les autres arts libéraux, était le fondement de la culture et de l'enseignement. Avec l'appui d'analogies grammaticales nées de la comparaison de la voix chantée (vox confusa, plus tard vox diastematica) et de la voix parlée (vox articulata, plus tard vox continua), et à l'aide du vocabulaire décrivant les formes et la fonction des accents grammaticaux, se développa une description verbo-grammaticale accentuelle des variations mélodiques, qui, du VIIème au XIème siècles, fournit une première terminologie. ${ }^{11}$ Ces mêmes accents grammaticaux latins inspirèrent, en partie tout au moins, un moyen mnémotechnique graphique empirique qui venait à l'aide du discours verbal et du geste : les neumes. ${ }^{12}$

\section{2) L'aide-mémoire des neumes}

Suivant les conceptions musicales modernes, les neumes peuvent être définis, quelles que soient leurs formes multiples, simples ou complexes, comme des signes écrits représentant un son, ou plusieurs sons non dissociés, dans un dessin mélodique évoqué graphiquement au dessus du texte à chanter. Je n'ai pas à parler ici de l'origine des neumes qui tient sans doute à la convergence de différentes influences - à côté de l'influence certaine des accents grammaticaux que je viens de mentionner et des signes ekphonétiques byzantins, placés au-dessus des parties les plus importantes du texte à cantiller, on a proposé celle des signes synthétiques de la cantillation talmudique, de certains signes prosodiques latins, etc - ni de l'époque de leur 
apparition (IXème siècle ?), ni de l'antériorité des neumes-points ou des neumes-accents, etc. Ce qui est important, du point de vue épistémologique que j'examine ici, c'est que, dans les conditions musicales et culturelles de l'exécution du chant grégorien aux VIIIème et IXème siècles, l'élaboration du système des neumes $a$, pour exprimer le mouvement mélodique de la voix dans une représentation spécifiquement musicale, réuni deux pratiques indicatives, deux formes d'indication d'action antérieures : l'une gestuelle, le signal chironomique de la tradition orale de la musique, suggérant la démarche continue du geste vocal, l'autre linguistique, le signe grammatical fragmenté de l'accentuation latine écrit sur le livre du Cantor. ${ }^{13}$ En effet, d'une part, bien que n'étant pas le résultat de la projection de la chironomie sur le parchemin, comme on l'a cru longtemps, ${ }^{14}$ les neumes sont, selon l'heureuse expression de Don Cardine, des " gestes écrits ", pour lesquels "le ductus de la plume a suivi le ductus de la voix" (1957 : 28 et 1970; également s.d. : 2) ; d'où le caractère indicatif imitatif et participatif des neumes : le geste "montre " ce qu'il faut faire, dans une action partagée par l'enseignant et l'enseigné. D'autre part, il y a un parallélisme quasi-total entre la description verbo-grammaticale des variations mélodiques de la voix et la notation neumatique, toutes deux étant des représentations phénoménales et empiriques issues de la pratique, résultant de la prise de conscience de la motricité vocale par intériorisation des actions sensorimotrices ${ }^{15}$ : ainsi, les indications d'exécution des pièces liturgiques dans la Musica disciplina d'Aurélien de Réomé (milieu du IXème siècle) semblent une description de neumes (1975:118-28). ${ }^{16}$

Cette notation se manifesta d'abord par l'addition de quelques neumes, puis de quelques lignes, et ne se trouve que tardivement sur des livres de chant entiers. Système complet et cohérent de signes, dont la forme et la disposition rendent compte de la nature et de la succession des sons, elle donne de la mélodie, tant dans ses variations de hauteur que dans son déroulement temporel, son rythme et son accentuation, une image graphique assez concrètement ressemblante. Intuitivement visualisés (la spatialisation étant nécessairement liée à cette visualisation), les neumes bénéficient des avantages des signes écrits qui, Saint Augustin l'avait appris aux clercs, ${ }^{17}$ conservent les sons et les paroles qui passent et les présentent à la vue : selon les termes de Solange Corbin, ils " parlent aux yeux ». Rompant le continuum vocal tout en le suivant, les 
neumes représentent (c'est-à-dire rendent présentes) sur le texte sacré les articulations du chant; ils suggèrent les formules grégoriennes connues, et ils évoquent directement la musique sensible en agissant sur l'imagination audio-visuelle, grâce à une certaine analogie avec les mouvements de la voix dont ils permettent une appréhension spatio-temporelle directe. Mais cette évocation, que l'immédiateté de sa saisie rend presque naturelle, est rudimentaire : ses indications directives sont très imprécises; les formes des signes sont différentes selon les régions et les époques, leur spatialité est souvent irrégulière et non directionnelle ; et, bien que favorisant la sensation de l'intervalle musical et l'image de la hauteur de son, la neumation n'indique pas leur valeur : au XIème siècle, Gui d'Arezzo compare les neumes sans repères de hauteur à "un puits sans corde $",{ }^{18}$ mais dès la période de grande diffusion des neumes à la fin du IXème siècle, des théoriciens comme Hucbald déplorent déjà cette imprécision (1784: 117b; cf. aussi, Odon de Cluny $1784: 252 b$ ). Expression de seconde main, car, traduisant une parole et un geste non musicaux, la neumation était plus un aide-mémoire qu'une instruction; son rôle mnémotechnique était limité, ${ }^{19}$ puisque son imprécision nécessitait la connaissance antérieure de ce qu'elle évoquait. La priorité du souci pédagogique réclama des moyens de mémorisation et d'enseignement plus efficaces que ceux de cette transmission que l'on pourrait appeler semi-orale, moyens qui puissent aller au-delà de la description verbale graphique empirique et de la répétition magistrale.

La notation neumatique correspondait bien à l'état d'âme des chanteurs de cette époque, à l'attitude du Cantor, pour lequel la musique devait être au second plan par rapport au texte sacré qu'elle servait. La réflexion musicale allait venir d'ailleurs : des rapprochements que pouvait faire le clerc, le Musicus, qui copiait, dans le cadre du Quadrivium, les développements de la cosmologie arithmo-musicale pythagoreo-platonicienne transmise de la culture antique (à côté des textes augustiniens inspirés par elle, les commentaires latins du Timée, l'œuvre de Martianus Capella) et, plus tard la théorie musicale grecque latinisée par Boèce. Les clercs musiciens du IXème siècle et leurs successeurs s'adressèrent à ces textes qu'ils adaptèrent à leurs problèmes en même temps qu'ils prenaient conscience de leurs problèmes à travers eux (donc en les modifiant, car il s'agissait de situations musicales bien différentes). Réunissant les préoc- 
cupations et les connaissances livresques et pratiques du Musicus et du Cantor, qui, dans la vie monachique et ecclésiastique, pouvaient être réunis dans un même individu - il est probable qu'Hucbald s'arrêtait de copier Chalcidius ou Boèce pour chanter les Vêpres - ils purent satisfaire, dans ce qui leur était parvenu de l'héritage musicographique antique, l'exigence de rationalité mnémonique et symbolique que l'insuffisance de leurs essais empiriques avait rendue nécessaire. Et, comme les résultats de spéculations heureuses sont souvent plus importants que ceux de l'expérience (Popper), c'est le Musicus qui va dominer le Cantor : celui-ci qui, avec la seule aide de sa mémoire et des neumes, s'orientait directement dans la musique, et sur lequel avaient reposé l'expressivité du texte sacré et l'âme du chant, perdra une partie de son savoir empirique et de sa perception finement différenciée; la pensée rationnelle remplacera ceux-ci par une notation précise, mais durement assujetissante, et le relèguera au rang d'exécutant docile et sans initiative : la « bestia » que dénonce au XIème siècle Gui d'Arezzo (1784b : 29).

\section{NOTATION ET THÉORIE : L'ÉCRITURE MUSICALE REPRÉSENTA-} TION DU DISCOURS THÉORICO-MUSICAL

\section{1) Nécessité d'une représentation mentale théorique intermé- diaire entre la musique et sa notation}

En effet, cette mémoire rationnelle, cette "mnémotechnie bien organisée " qui doit nous permettre de retrouver le fait (Bachelard 1949 : 44) et qui, par la compréhension et la fixation d'un petit nombre de principes descriptifs rationnels (intervalles consonants, échelle, modes) enchaînant de façon systématique et cohérente les faits musicaux, suffit pour en reproduire un très grand nombre, ne pourra être fournie aux chantres du haut Moyen-Âge que par la théorie musicale. ${ }^{20}$ La description verbale de la mélodie avait déjà permis (avec l'intervention d'autres influences que je n'ai pas à considérer ici) d'édifier un ensemble de règles pratiques, faciles à retenir, pour assembler et réassembler les formes musicales globales, les formules vocales (inflexio vocis) qui constituent le continuum sonore: en les réduisant qualitativement à quelques formules types (formules apéchématiques, formules de récitation), et en les classant ordinalement avec les pièces correspondantes dans les Tonaires pour pouvoir les enchaîner de façon euphonique, ce véritable 
"stratagème de la mémoire » (ibid : 32) formait une pré-théorie empirique du chant modal grégorien, que complétait la notation neumatique appartenant à la même sphère de pensée musicale (cf. Duchez 1979 : 66-67). Mais cette modalité formulaire qui, reprise dans le cadre de la rationalisation quantitative de la musique, deviendra la théorie de la modalité grégorienne, n'offrait sous sa forme empirique que des possibilités combinatoires restreintes ; et, comme sa notation, elle s'avèra vite opérationnellement insuffisante pour la pratique du chant. Une notation rationnelle, supprimant l'effort de conservation de la mémoire et réduisant celui de la transmission pédagogique, ne fut possible que dans et par la rationalisation théorique : de même que la mémorisation de la musique fut le but de la recherche d'une notation, aide-mémoire symbolique, de même la recherche de la notation musicale fut le but et le résultat de l'élaboration d'une rationalisation théorique de la musique, aide-mémoire logique. À la naissance de la théorie musicale occidentale, qu'il s'agisse de la notation de la hauteur ou de celle plus tardive de la durée, mémorisation, rationalisation, notation sont trois questions qui se provoquent, se rencontrent, se complètent et se résolvent mutuellement. La notation qui résulte de la théorie est aussi l'outil de son développement et les acquisitions de la notation correspondent à des acquisitions théoriques. La cohérence rationnelle de la théorie engendre la mnémotechnique rationnelle de la notation correspondante. Théorie et notation se déterminent l'une l'autre, et, aux siècles que nous étudions, il est impossible de séparer leur histoire intriquée.

Il faut insister sur le fait que la notation à laquelle va aboutir cette double histoire ne symbolisera pas le phénomène musical lui même, comme avait tenté de le faire la notation neumatique ; elle ne représentera pas la musique elle-même, mais le système de concepts qui, lui, est élaboré pour représenter la musique. Les signes de la notation correspondent à des rapports d'ordre et de mesure, à des relations conçues, fixées et formulées mathématiquement, instaurées par la théorie. La notation musicale occidentale traditionnelle, qui a pris naissance aux IXème-XIème siècles, c'est la théorie musicale occidentale traditionnelle, qui a pris naissance aux IXème-XIème siècles. Et tout au long de son évolution, cette notation représentera le système de représentation, la théorie, dont elle est issue ; elle ne pourra s'adapter de façon adéquate (avec des modifications évolutives) qu'à des musiques basées sur les principes théoriques fondamentaux qui 
lui ont donné naissance et qui la sous-tendent : d'où son inefficacité quand ces principes deviennent caduques, et ses difficultés, sinon son incapacité, pour noter des musiques étrangères à la tradition théorique occidentale, qu'elles soient exotiques ou nouvelles. ${ }^{21}$ La théorie musicale, fondant la notation qui l'exprime, nous donne les moyens nécessaires pour en déchiffrer l'information transmise (apprendre et assimiler notre notation et son enchaînement d'automatismes se dit d'ailleurs apprendre « la théorie ", et apprendre à composer la musique en appliquant la théorie se disait encore récemment apprendre "l'écriture ") ; et la notation traditionnelle nous est facilement et culturellement transparente, au contraire des neumes qui ne correspondent pas à une rationalisation préalable, et même, plus tard, au contraire de la notation mesurée de la durée qui correspond à une rationalisation incomplète, ne possèdant pas cette solidarité systématique des concepts qui fait la prégnance de notre théorie des hauteurs. ${ }^{22}$

Ce sont les conditions historico-musicales de l'élaboration de la notation qui ont imposé la nécessité d'une représentation intermédiaire entre la musique et sa représentation graphique. En effet, d'une part l'objectivation du phénomène musical, et la prise de conscience de l'autonomie des structures musicales à noter, étaient particulièrement difficiles dans le cas de la liaison existentielle et phénoménale du texte verbal et de l'élément sonore musicalisé, qui caractérise le chant grégorien; et les neumes ne notaient pas la musique accompagnant le texte, mais guidaient la voix prononçant le texte et par rapport à lui. D'autre part, dans ce monde médiéval encore sans technique (le monocorde est venu plus tard), la voix nue n'offrait aucun repère physique fixe et aucune référence pratique, permettant d'établir un système de signes couvrant psychologiquement les modalités concrètes de la production du son. Ces repères et ces références sont naturellement offerts dans la musique instrumentale par le fonctionnement de l'instrument (ainsi les cordes de la lyre dans la théorie grecque dont les médiévaux ont une certaine connaissance, ou les tuyaux et les claviers d'orgues sur lesquels ils signalent par la série des lettres alphabétiques le résultat sonore des actes instrumentaux) ; et ils permettent toujours l'établissement de tablatures indiquant concrètement sur l'instrument, ou sur le schéma qui le représente, le geste humain à exécuter. Mais, comme l'a fort justement remarqué Hugo Cole (1974 : 37 41), le chanteur, lui, n'a pas de guide physique mécanique, et il 
doit, avant de produire un événement sonore (son, intervalle, formule), l'identifier dans son imagination. Cette identification il peut la faire plus ou moins facilement grâce à la mémoire immédiate de la transmission orale, aidée par les neumes; il la fera plus tard beaucoup plus aisément par l'intermédiaire d'une série d'évaluations rationnelles des relations sonores, évaluations possibles grâce à l'abstraction et à la conceptualisation que lui apportera la théorie. Dans un sens, les neumes, indicateurs du geste vocal, essaient d'être une sorte de "tablature pour la voix", sans avoir les possibilités matérielles d'atteindre la précision gestuelle analytique et l'évidence efficace de la tablature instrumentale. Ces difficultés spécifiques de repérage et de référence se font bien sentir dans la terminologie incertaine et embarrassée de la description verbale de la mélodie; ainsi la description du rapport mélodique entre la dernière note du Verset et la première note de l'Antienne à réentonner, ou la description des formules de récitation de la psalmodie, par Aurélien de Réomé (1975 : chap. 10-14 et 19).

Il apparaît donc qu'une notation, indication symbolique toujours présente, ne puisse représenter la musique vocale absente, phénomène concret mais dépourvu de repères appartenant au monde physique observable et mesurable, sans l'existence, entre le phénomène et l'indication, d'une représentation mentale de nature représentative différente, d'un système conceptuel donnant du phénomène une description théorique, dont les caractéristiques et les relations abstraites sont formalisables en un système symbolique. Pour noter la musique, on ressentit donc peu à peu et inconsciemment (ainsi, on ne trouve pas une conscience claire de cette nécessité dans Hucbald, alors qu'elle est exprimée avec précision par Jean Scot Erigène) ${ }^{23}$ la nécessité d'une description préalable : une représentation théorique, non de l'opération productrice du chant trop difficile à décrire dans ces conditions, nous l'avons vu, mais du résultat hypothétique qu'on voulait réaliser, les sons et leurs relations. ${ }^{24}$ A cette représentation intermédiaire, la théorie musicale, revenait la tâche de déterminer ce qu'on voulait représenter abstraitement pour reproduire ou produire ce résultat sensible, et de trouver les moyens discursifs conceptuels pour cette description. Cette description théorique pourra être retraduite en indications pratiques d'opérations concrètes, au moyen de sa représentation graphique, la notation, sur laquelle on effectuera les opérations mentales mises au point grâce à la théorie. Ainsi, avant de modi- 
fier la pensée musicale par le développement technique qu'elle permet, la notation exigea une organisation théorique de cette pensée, donc déjà une modification importante de celle-ci. Cette réorganisation, cette théorie, va, pour élaborer la possibilité de penser le phénomène musical, le décomposer analytiquement en relations fonctionnelles entre éléments paramétriques, en s'appuyant sur l'héritage musico-culturel antique et sur l'élucidation des démarches musicales empiriques antérieures qui seront, grâce à elle, dépassées. Elle va créer ainsi un nouveau mode d'accès à la réalité musicale sensible, un mode rationnel abstrait moins évident intuitivement que les descriptions verbales et neumatiques de la musique, dont les seules ressources étaient les métaphores gestuelles et les analogies langagières (grammaticales et accentuelles). Mais elle ouvre le monde infini des techniques opératoires de combinaison et des synthèses inductives, dont la notation, porteuse de ce nouveau mode d'appréhension de l'univers sonore, et ayant conquis par le détour de la théorie son pouvoir opérationnel et synthétisant, va permettre les réalisations.

\section{2) Aperçu sur les caractères sémiologiques de la représenta- tion vocale graphique qui guidèrent la recherche théorique \\ L'étude des démarches par essais et erreurs qui, du IXème au} XIème siècle, des neumes à la portée, ont élaboré les bases fondamentales et perdurables de la notation occidentale traditionnelle, relèvent donc, d'après ce qui précède, plus de l'épistémologie de la représentation conceptuelle de la musique ${ }^{25}$ que de l'épistémologie du signe (et de la sémiologie). Il faut cependant dire rapidement quelques mots sur les conditions sémiologiques de la notation qui va se fixer pendant cette période. On ne peut que suivre Isidore de Séville (et les ethnomusicologues seront sans doute d'accord avec lui) lorsqu'il affirme qu'on ne peut pas " écrire les sons "; en effet, on peut tout au plus les "décrire", soit, nous l'avons vu, par la description de leurs conditions concrètes de production, montrant ainsi à l'interprète ce qu'il a à faire : c'est ce qu'on a tenté de faire par les neumes ; soit, et c'est ce qu'a fait la notation classique sur portée, par le détour théorique de leurs caractéristiques paramètriques abstraites qui n'en donnent que certains éléments, choisis pour l'importance et la pertinence de leur rôle structural dans le fait musical étudié, et d'après l'état de nos connaissances rationnelles concernant ce fait. Quoi qu'il en soit, le rapport entre le signe et le son est, selon 
l'expression du compositeur Franco Donatoni, problématique ${ }^{26}$ et chaque notation entretient avec sa musique des rapports spécifiques, suivant le type de communication qu'elle cherche à établir.

Si la connaissance des conditions musicales et intellectuelles d'élaboration, au IXème siècle, est indispensable pour la compréhension de la forme et du fonctionnement de notre notation musicale traditionnelle, il n'est pas inutile, pour comprendre le sens des premières démarches de cette élaboration, de regarder en avant vers son avenir et sa fixation définitive, selon les connaissances sémiologiques actuelles. D'après les analyses des spécialistes de la communication, ${ }^{27}$ on peut déduire que ce qui s'élabore alors est un code indirect de substitution, correspondant à un système de concepts qui s'articule entre le signe et le sens. Et, du point de vue formel, la notation musicale traditionnelle à laquelle aboutira cette élaboration, est, comme l'écriture du langage, un système cohérent et hiérarchisé de signes discrets, ${ }^{28}$ récurrents et structurés, possédant une structure temporelle syntagmatique dans laquelle chaque unité est en relation avec celles qui précèdent et celles qui suivent; comme l'écriture langagière, elle est une représentation visuelle et durable, conservable et transportable, qui analyse l'expérience musicale concrète de façon successive et linéaire. En tant que code d'information, elle répond aux exigences qu'énonce avec raison $\mathrm{Nel}$ son Goodman pour tout système de notation musical (musical notational system) : mise en relation de dénotation entre des caractères syntaxiquement et sémantiquement disjoints et différentiels (cf. Goodman 1968 : 177-82). A ces conditions sémiologiques générales, la recherche musicale notationnelle ajoute une exigence spécifique concernant la facilité et la rapidité de lecture et d'exécution : la nécessité de donner à la musique une représentation visuelle qui soit en corrélation perceptive avec son audition orale.

Pour passer de la représentation phénoménale analogique qualitative et intersubjective du IXème siècle, à la représentation théorique, scientifique quantifiée et objective, même dans sa forme primitive, au XIème siècle, la recherche notationnelle va devoir déterminer son signe musical discret, préciser l'espace musical graphique dans et par lequel il fonctionne, opérer la systématisation des signes dans cet espace, quantifier et proportionaliser les relations paramétriques que représentent ces signes. D'abord, le signe musical est à la fois phonique (il se 
prononcera au XIème siècle par les syllabes guidoniennes) et graphique : c'est la note de musique qui unit un signe visuel, une image acoustique et un concept théorique ; c'est un signe discret - le continuum sonore sera rompu en sons discrets, l'unité distinctive du son correspondant à l'unité graphique de la note qui fixe la discontinuité de la pensée musicale, ce qui assure et augmente ses possibilités de combinaison. Il n'est pas arbitraire (quoi qu'on en ait dit) au contraire de tant de signes graphiques : pour succéder aux neumes figuratifs et ressemblants de la description verbale de la musique, on fera correspondre sa forme et sa disposition au concept qu'il représente et qui exprime scientifiquement le caractère sensible pertinent qu'il note : la hauteur ; et son absence d'arbitraire, facilitant l'exercice de la mémoire immédiate sensible qui opère la sommation des signes, jointe à sa rationalité, facilitant l'exercice de la mémoire médiate logique, augmente l'économie mnémonique du signe. Il est systématisé en " un système d'unités bien définies se combinant et se structurant suivant des règles bien définies ${ }^{23}$; et ce système de signes ne correspond pas de façon directe à la réalité musicale empirique, mais au système de sons qu'a élucidé la théorie musicale, et dont il a le même niveau d'organisation : cette organisation avait été qualitative pour les neumes, ordinale pour la notation alphabétique, elle est quantitative et métrique pour la notation traditionnelle sur portée. Pour cette représentation quantitative, le signe musical traditionnel, consistant en points et lignes, utilise les propriétés géométriques et métriques du plan, y inscrivant en hauteur la valeur figurée du paramètre alors existentiel du son, que la théorie a abstrait du continuum sonore, la hauteur ; et employant la largeur pour le déroulement temporel de la musique " émise et perçue sur la trame même du temps" (Mounin 1970:72) : remplaçant la spatialisation naïve des descriptions verbo-gestuelle et grammaticale (image spatiale réalisée dans la chironomie, les accents et les neumes), la spatialisation rationnelle de la théorie crée un espace de représentation, cadre de mesures numériques et de proportionalité : et celui-ci sera projeté en un espace symbolique graphique, un espace notationnel où les signes musicaux auront des positions relatives variant systémiquement avec les paramètres sonores. L'espacement inhérent à toute écriture, et déjà présent dans le monde des neumes mais avec des conséquences assez limitées (dont on essaie d'ailleurs d'expliciter le sens), exige pour une notation rationnelle, basée, nous l'avons dit, sur des disjonctions 
et des différenciations, cet espace de représentation théorique. D'autre part, la représentation théorique de la musique requiert cette spatialité, directement liée à nos schémas d'existence biologique (Bergson), conforme aux tendances profondes de notre esprit, et qui constitue, avec le temps, le cadre conceptuel nécessaire de toute connaissance de la réalité physique et de sa description. L'espace théorique de représentation musicale fut d'abord un espace de séparation et de différenciation où se déploient les distances intervalliques, où s'espacent les sons discrets qui étaient indistincts et soudés dans les formules, où se détermine conceptuellement et quantitativement le paramètre de hauteur du son ; il deviendra très vite un espace d'organisation et de construction où se développe l'échelle musicale quantitative, où s'échelonnent les modes grégoriens, où se disposera la polyphonie. ${ }^{30}$ Et le pouvoir de différenciation, d'orientation et d'organisation de cet espace musical théorique est à la fois précisé et amplifié par la représentation graphique qui s'y déploie, avec sa portée scalaire étagée verticalement et déroulée horizontalement, et qui peut recevoir et intégrer un nombre considérable, sinon infini, de combinaisons sonores correspondant aux cadres de l'espace théorique qu'elle a exigé et qu'elle exprime.

\section{THÉORIE ET MUSIQUE : LE DISCOURS THÉORICO-MUSICAL INTERMÉDIAIRE ENTRE LA MUSIQUE ET SA NOTATION}

\section{1) Objectivation, abstraction conceptuelle et quantification des phénomènes sonores du chant grégorien}

Tous ces concepts théorico-musicaux de caractère spatial ou de fonctionnement spatial ont fondé la notation dont le but était de les représenter ; ils ont permis de passer de l'appréciation verbale qualitative du mouvement de la voix dans la pronuntiatio liturgique du discours sacré, symbolisée graphiquement par les neumes, à l'évaluation quantitative des intervalles entre les sons et à leur mensuratio numérique, préfigurée par la notation sur portée qui, avec sa clef, représente l'échelle des sons devenus autonomes. Ces concepts spatiaux, mesurables et représentables graphiquement, ont été élaborés grâce à l'introduction et à l'adaptation de méthodes et de notions musicales quantitatives transmises de la théorie musicale grecque (théorie pythagoricienne des intervalles consonants, mesures au monocorde). 
Cette élaboration se réalisa du IXème au XIème siècle, du De octo tonis attribué autrefois à Alcuin et de la Musica disciplina d'Aurélien de Réomé (ca. 840-849), qui donnent encore une description verbale et neumatique de la mélodie, à l'oeuvre de Gui d'Arezzo (entre 1028 et 1032) et de ses disciples, où s'effectua la grande synthèse théorique de l'échelle, de la modalité et de la notation, qui apportent ensemble et complémentairement une solution complète au problème de la mémorisation du chant liturgique. Cette édification conceptuelle - changement complet d'outillage mental grâce auquel la connaissance remplace, dans la saisie de la musique, le découpage empirique qualitatif en formules mélodiques, données naturellement par la portée du souffle, par un découpage rationnel en éléments quantitatifs, mesurés techniquement au monocorde (intervalle, hauteur relative du son, échelle musicale) - s'amorce au milieu du IXème siècle dans les Commentaires carolingiens de Martianus Capella (Jean Scot Erigène, Martin de Laon et anonymes). ${ }^{31}$ Elle se poursuit à la fin du IXème et au début du Xème siècle dans les traités de musique mathématisants des premiers lecteurs de Boèce (Réginon de Prüm, Rémy d'Auxerre, et surtout Hucbald; les traités anonymes de l'Alia musica, et surtout de la Musica Enchiriadis et ses Scholies). Elle s'accomplit durant le Xème siècle, grâce à l'assimilation du De Institutione musica de Boèce, dont les méthodes fécondes de mesures expérimentales au monocorde, d'échelonnement sonore (échelle des sons et échelle des tons) et de notation systématisée (Livre IV), complétèrent de façon opérationnelle et pratique le fond de connaissances théorico-musicales plus spéculatives et moins techniques que les gens du Moyen-Âge avaient jusque là reçues de l'Antiquité. ${ }^{32}$ Mais malgré l'aide de l'héritage de la pensée antique, cette élaboration théorique et notationnelle exigea un effort considérable d'objectivation des phénomènes sonores et d'abstraction conceptuelle.

Le travail d'abstraction lent et obstiné qui s'effectua tout au long des IXème-XIème siècles, a permis, par division conceptuelle, que la pensée discursive accède à la globalité du fait musical : dans un double mouvement mental entre l'expérience musicale concrète du chant liturgique et le savoir arithmomusical abstrait antique du Quadrivium, il détache de la réalité musicale senso-affective, des notions générales et des caractères variables sur lesquels l'esprit pourra opérer. Les notions musicales et les caractères différentiels dégagés, d'une part sont 
adaptés, avec plus ou moins de pertinence des connaissances théorico-musicales de la transmission antique ; d'autre part, ils sont en même temps choisis par la perception (qui exerce ellemême un début d'abstraction) à partir d'un point de vue dominant dans cette perception : ce choix laisse donc en dehors de la théorie musicale et de la notation une partie moins nettement perçue du phénomène vocal (le timbre, l'attaque), qui disparaît pour des siècles de la pensée musicale consciente et rationnelle. Le mouvement d'abstraction, comme la description verbale qualitative et neumatique à laquelle la perception avait déjà fourni les mêmes critères sonores, part de l'intériorisation des actes sensorimoteurs concrets du chanteur ; mais cette fois l'intériorisation s'effectue dans les cadres logico-mathématiques ${ }^{33}$ que les penseurs médiévaux ont acquis de la culture antique, et qui leur permet de mener jusqu'au bout la conceptualisation abstraite. Et cette conceptualisation abstraite va permettre de substituer le signe au concept, donc de noter, et ainsi de substituer la déduction à l'expérience.

L'abstraction des concepts musicaux exigeait d'objectiver les phénomènes musicaux en tant que tels, c'est-à-dire de séparer dans la conscience (séparation qui fut tardive) la musique chantée de l'action de chanter. Les premiers stades pratiques de cette objectivation avaient été le geste chironomique, le classement formulaire, les signes neumatiques; le dernier stade fut théorique. L'objectivation, qui passe par la saisie de l'importance de l'aspect physique des phénomènes sonores, et aboutit à leur description précise et à leur symbolisation graphique, fut définitivement réalisée, au Xème siècle, grâce à l'emploi du monocorde, véritable outil musical qui permettait la mesure des intervalles selon la théorie pythagoricienne des rapports de consonance : en faisant correspondre un phénomène physique audible (son, intervalle) à la production mécanique qui le réalise et à la mesure numérique des facteurs de cette production (longueurs de corde), le monocorde détacha définitivement le fait musical de sa condition langagière, de son intégration au texte du chant et de sa description verbale et neumatique, pour l'engager pratiquement dans la conception mathématique des phénomènes musicaux transmise de l'Antiquité; en même temps, il l'orientait définitivement vers la conception scientifique expérimentale, impliquant l'observation auditive et la mesure mathématique qui, dans l'avenir, liera la connaissance théorique de la musique à celle de ses corrélats acoustiques 
quantitatifs. Le monocorde a en effet permis aux musiciens médiévaux de construire pratiquement la " quantité musicale ». L'introduction de la mesure, aboutissement de la réflexion objective qui fait du nombre la propriété intrinsèque de l'objet mesuré, a permis de joindre à l'appréhension qualitative de la perception le pouvoir de la connaissance des " raisons » quantitatives. ${ }^{34}$ Les mesures monocordales donnaient une description numérique fine et exacte des phénomènes musicaux qu'elles isolaient, dans un nouveau langage bénéficiant « de la clarté, de la précision et de l'universalité traditionnellement reconnus à la langue mathématique " (Bachelard 1928: 52). La description monocordale quantitative se rattachait idéologiquement à la foi profonde qui impliquait la musique dans une vision cosmomathématique du monde, et au principe théorique efficace de la proximité ontologique de la musique et des nombres : foi et principe qui ont fondé la théorie musicale grecque, et qui, partagés par le Musicus médiéval, ont dirigé et facilité la quantification de la musique liturgique occidentale, malgré ce que cette quantification avait de brutal pour l'inflexion grégorienne. ${ }^{35}$ Cette description monocordale faisait désormais correspondre la structure d'un ensemble de phénomènes sonores à des opérations réelles, conformes à la structure d'un ensemble de nombres correspondant à des opérations arithmétiques ; elle établissait ainsi un ordre mathématique dans le tissu enchevêtré des phénomènes musicaux, ordre intellectuel bien différent du réalisme qualitatif et de l'intuitionnisme grammatical qui régissaient l'approche de la musique auparavant. Et cette description monocordale réalisait ainsi les conditions sonores, visuelles et mathématiques de l'élaboration de la notation rationnelle, vers laquelle tendaient les recherches notationnelles depuis le début du IXème siècle, notation pouvant représenter quantitativement (ce qui est la représentation la plus simple, biens que non la plus directe), des relations paramétriques fonctionnelles; de plus le monocorde offrait, nous le verrons, grâce à ses reprères numériques indiqués par les lettres de l'alphabet, un moyen précis, bien que peu opérationnel pour la pratique du chant, de noter alphabétiquement les éléments théoriques abstraits du continuum vocal.

2) Résultats théoriques et notationnels de l'objectivation quantitative et de l'abstraction conceptuelle

Ce travail d'objectivation quantitative et d'abstraction con- 
ceptuelle aboutit à l'édification des cadres conceptuels de la représentation théorique et graphique de la musique; cadres conceptuels qui, j'ai déjà eu l'occasion de le dire, furent pour un millénaire transformés en cadres perceptuels, la perception s'exerçant à travers la grille des catégories et des notations de la théorie. Citons rapidement ces principales acquisitions conceptuelles-perceptuelles quantifiées qui n'ont pas été élaborées en même temps, mais qui n'ont pris leur plein sens que dans leur synthèse et leur systématisation.

1. L'intervalle, première notion perceptive dégagée du continuum vocal formulaire; notion qui réunit, en gros les notions de mouvement de la voix, de distance dans l'espace sonore, enfin de rapport numérique abstrait concrétisé au monocorde. Il est le premier élément de constitution de l'échelle musicale et de différenciation des modes, une importance spéciale étant donnée à la notion de ton comme unité intervallique scalaire. Et il s'intègre facilement dans l'espacement de l'écriture musicale qu'il a contribué à créer.

2. Le son discret plus tardivement isolé que l'intervalle dans le continuum vocal formulaire (au contraire du son instrumental); unité sonore minimale, il fut, en raison de son origine graphique grammaticale et neumatique (cf. Duchez 1981 : 569-70), tardivement objectivé comme valeur quantitative. Cette valeur quantitative qui donnera son existence théorique au son musical et permettra de la noter, c'est

3. La hauteur de son, abstraction du caractère sensible pertinent grave-aigu, valeur typiquement discrète et mathématiquement simple correspondant à une "différence quantitative distinguée de toutes les autres "; c'est une notion occidentale construite assez tard à partir de la description qualitative verbale et neumatique et des concepts quantitatifs de la théorie grecque qui cependant, basée sur l'idée de tension, ignorait cette notion. Pendant des siècles, le son, considéré théoriquement comme un unité abstraite, support permanent de relations variables, sera caractérisé par sa hauteur; on lui ajoutera progressivement d'autres paramètres (durée, intensité), qu'exprimait auparavant la notation neumatique du mouvement, vocal, mais ce seront des additions modifiant ces qualités sans changer sa nature théorique. ${ }^{36}$ 
4. La note de musique, signe graphique du son conceptualisé et quantifié par sa hauteur, et dont la notion date du milieu du IXème siècle, ${ }^{37}$ sera l'élément expressif et opératoire primordial de la notation discrète qui caractérise la musique occidentale. Résultat d'une série de conceptualisations, la note de musique conditionnera la perception conceptualisée pendant plus de dix siècles (elle correspond en effet épistémologiquement à l'arithmétique et à la géométrie grecques transmises au Moyen Âge, et à l'acoustique de la mécanique classique). Limitée alors à ce que la théorie retient du son phénoménal, la hauteur, on la changera plus tard de valeurs de durée, en lui adjoignant des modifications de forme correspondant aux théorisations ultérieures de la durée (temporalisation facilitée par le déroulement temporel de la notation). Dans l'expression de la durée comme dans celle de la hauteur, mathématisation des deux aspects élémentaires de la musique, ses propriétés spatiotemporelles, la note discrète analyse l'expérience mélodique globale en éléments successifs articulés sous forme linéaire.

5. La notion d'échelle musicale enfin, qui résulte d'une part, de l'aboutissement des courants qui élaborèrent les quatre concepts précédents et des méthodes monocordales qui les quantifièrent selon l'accord pythagoricien; et d'autre part, des influences conjuguées des diagrammes de la musique grecque exposés par Boèce et des gammes planétaires de la doctrine cosmologique arithmomusicale de l'Harmonie des Sphères, répandue au Moyen Âge par le pythagorisme néoplatonicien. L'échelle n'a pu être objectivée que par la représentation graphique qui l'implique et la manifeste : sa notation spatiale sur la portée construite par tierces, qui est, comme elle, contraignante.

La synthèse théorico-musicale de la notation sur portée, réalisée au début du XIème siècle par Gui d'Arezzo (je n'ai pas à discuter ici de l'exactitude de cette attribution), put s'effectuer grâce à l'interaction dialectique entre la construction théorique et la recherche notationnelle : la logique de l'écriture provoque, comme l'a bien montré Georg Von Dadelson (1964 : 17-25), une prise de conscience des éléments logiques de la musique, et ceux-ci aboutissent eux-mêmes à la construction logique de la notation. Mais durant les deux siècles pendant lesquels se déve- 
loppait cette théorie et cette notation, d'un côté les neumes poursuivaient leur rôle d'aide-mémoire, participant au courant dialectique rationnel théorie-notation par l'accroissement de leur caractère diastématique dans de multiples formes évolutives (ils seront d'ailleurs finalement placés sur la portée, gagnant la précision de hauteur au prix d'une grande perte sémiologique dans l'expression des autres éléments musicaux) ; d'un autre côté, se développèrent différentes notations de rationalité plus ou moins poussée, liée à certains aspects du développement théorique, qui furent finalement supplantées par la notation sur portée. Tous ces essais et échecs sont extrêmement éclairants sur le fonctionnement de la notation et le rapport entre la notation et sa musique.

\section{NOTATION ET MUSIQUE : LES FONCTIONNEMENTS NOTATION- NELS ET LES RAPPORTS NOTATION - MUSIQUE}

\section{1) Stades notationnels intermédiaires entre les neumes et la portée}

La rationalisation graphique des neumes qui introduisit des habitudes signalétiques préparant la portée diastématique, est dûe en partie aux nécessités de la représentation des phénomènes physiques dans le cadre espace-temps, graphisme qui exige, nous l'avons vu, l'utilisation des deux dimensions du plan. Bien que les neumes aient le plus souvent recours à des moyens non graphiquement diastématiques pour exprimer les variations grave-aigu, ils contribuèrent cependant à la fixation du son dans l'espace sonore (cf. Kunze $1974: 6-7$ ) : principalement en liant la sensation d'intervalle musical à une image de distance spatiale (en particulier pour indiquer la distance intervallique entre la dernière note du verset et la première note de l'antienne à réentonner, ou par l'usage du custos). Selon Peter Wagner, les neumes auraient dès leur début possédé un caractère diastématique dû à la ligne gravée peu visible qui guidait les notatores, caractère accentué ensuite par l'évidence d'une ligne colorée; ce caractère diastématique est reconnu aux neumes paléo-francs et bretons, et il est ajouté aux neumes de Saint-Gall par certaines lettres significatives; il est tout à fait net pour les neumes aquitains du XIème siècle, ponctuels et superposés verticalement par espaces proportionnels aux intervalles; il est explicite pour les neumes sur lignes apparus à la fin du Xème 
siècle; et on a pu dire que les neumateurs étaient allés au devant de la diastématie (cf. Wagner 1912 : chap. XII et XIII).

L'effort de diastématisation se retrouve aussi dans les différentes notations qui ont été essayées entre la période préthéorique de la naissance des neumes, au IXème siècle, et l'élaboration complète de la théorie musicale mathématisée, au début du XIème siècle qui vit se répandre l'usage de la portée. Mon dessein n'est pas de faire ici l'étude historique de ces graphismes musicaux plus ou moins rationnels. Je mentionnerai seulement ces notations qui ont été très étudiées. En premier lieu chronologiquement, la notation d'Hucbald (fin IXème siècle), qui possède déjà la notion d'intervalle et d'échelle, et emprunte à Boèce le grand système parfait diatonique grec (et sa notation) sans bien comprendre les relations acoustiques qui l'ont engendré ; critiquant l'imprécision des neumes et l'enseignement oral de la musique, il propose (en sus d'une notation non diastématique, consistant à placer sur les syllabes du texte à chanter des lettres inspirées de l'exposition du trope lydien par Boèce et indiquant une hauteur fixe) une notation diastématique naïve :il place les syllabes du texte dans les interlignes diatoniques dont la valeur est précisée (tonus, tonus, semi-tonium, tonus), d'une sorte de portée de cinq lignes. Quelques années plus tard, la notation de la Musica Enchiriadis, basée sur la notion précise d'intervalle et d'échelle, correspond à une échelle par tétracordes notés par les quatre signes fondamentaux dasiens selon les quatre finales des tons liturgiques (ré, mi, fa, sol) : il est joint à cette échelle, destinée surtout à l'organum, une disposition diastématique consistant à placer les syllabes du texte sur les lignes d'une sorte de portée dont les hauteurs fixes sont indiquées par les signes correspondants de l'échelle dasienne servant de clefs. Ajoutons que ces notations diastématiques ont inspiré à Hermann Contract, pendant la réforme diastématique guidonienne du XIème siècle, une notation intervallique non diastématique, dans laquelle les intervalles sont indiqués sur le texte à chanter par des combinaisons de lettres (e pour l'unisson, $s$ pour le demi-ton, $t$ pour le ton, $d$ pour la quarte, $\Delta$ pour la quinte, le sens ascendant ou descendant étant indiqué par un point en dessus ou en dessous).

A côté du développement croissant de la diastématie, cette période d'actives investigations notationnelles vit l'emploi, limité mais de grandes conséquences, d'un moyen d'indication non diastématique des sons discrétisés : la notation alphabé- 
tique. Il faudrait consacrer à cette notation dont l'étude a été magistralement ébauchée il y a plus de vingt ans, ${ }^{38}$ une analyse systématique fouillée, tenant compte des connaissances actuelles de sémiologie générale, des nouvelles méthodes historiques et des récentes découvertes codicologiques. Je n'aborderai pas cette question malgré son importance, me contenant de dire que cette notation (conservée dans la nomenclature solfégique des pays anglo-saxons) est apparue dans la période d'intenses recherches théoriques : on rencontre l'emploi des séries alphabétiques pour représenter les sons discrets à partir de la fin du IXème siècle jusqu'à la fin du XIème siècle ${ }^{39}$ dans à peu près tous les traités de théorie et ouvrages didactiques, et dans certains livres de chant grégorien (ajoutée au texte préexistant pour note avec précision les nouveautés et les pièces peu connues); on le rencontre à partir du Xème siècle (malgré quelques allusions au IXème siècle) jusqu'au XIème siècle $e^{40}$ pour les mesures instrumentales, principalement celles des tuyaux d'orgues et les " alphabets » de leur clavier. On s'accorde généralement à voir l'origine de leur clavier. On s'accorde généralement à voir l'origine de cet usage dans le De institutione musica de Boèce, connu dans la deuxième moitié du IXème siècle : Boèce emploie en effet constamment les lẹttres de l'alphabet comme repères mathématiques et géométriques dans ses démonstrations acoustiques et les diagrammes correspondants (nombreuses séries différentes), ou comme moyens d'exposition dans ses descriptions des intervalles (en particulier dans la description des aspects d'octave) ; mais il emploie aussi l'alphabet comme un véritable système sémiologique déductif, pouvant, suivant le contexte, fonctionner comme une notation musicale : une première fois pour la division monocordale numérique du grand système parfait qui donne l'échelle grecque complète, grand diagramme copié pendant tout le Moyen-Âge sous le titre de Monocorde de Boèce ${ }^{41}$; une seconde fois pour l'échelonnement des sept tropes de hauteurs sur le grand système parfait, pour l'explication de leur fonctionnement et le diagramme correspondant, très imité au Moyen-Âge sous le nom de notation de Boèce. ${ }^{42} \mathrm{Il}$ a été aussi proposé pour la notation alphabétique médiévale une origine instrumentale plus ancienne, les tablatures d'orgues byzantines introduites au VIIIème siècle $e^{43}$; quoiqu'il en soit, les textes montrent que la notation alphabétique, est, pour le Moyen-Âge, liée à Boèce et au Monocorde. Son développement, dans de nombreuses séries différentes, aboutit au redoublement des signes à 
l'octave et à l'addition du Gamma $\Gamma$ au grave; sa diffusion fut limitée dans la pratique vocale par son impuissance à établir les groupements des sons et à exprimer les liquescences. Développement et diffusion résultent de la convergence de trois recherches théorico-pratiques : l'adaptation du grand système parfait diatonique grec au système des tons liturgiques, la « monocordisation » des repères d'échelonnement des tons et de leurs intervalles, et la cyclisation des sons selon l'octave qui, de consonance optimale dans l'antiquité, devint cadre structurel de l'échelle occidentale. L'enseignement du chant se faisant à partir du IXème siècle au monocorde (de façon quasi audiovisuelle), lorsque la division du monocorde fut systématisée en échelle, les repères monocordaux alphabétiques furent, l'exemple de Boèce aidant, systématisés en notation. ${ }^{44}$ Cette systématisation alphabétique des hauteurs de son a été facilitée par l'analogie grammaticale du son et de la lettre, répandue dans la culture grammaticale des siècles précédents et par la conception pythagoricienne qui liait le son au nombre (cf. Duchez 1981 : 569-70 et 577-78). Elle utilisait la capacité de sériation précise et déductive de l'alphabet : l'ordre de succession des sons suit l'ordre de succession des lettres, successions dont les éléments ne sont pas indépendants les uns des autres, un son, une lettre donnant la " place » des autres dans la série.

On a écrit non sans raison que "le développement de la notation moderne, de la notation neumatique à la portée, a été un processus si tortueux que tout résumé rapide oblige à dénaturer les faits historiques " (Cole $1974: 44)$. L'analyse épistémologique permet cependant de dégager, à partir des stades intermédiaires connus, les lignes de rationalité de ce développement, et de constater que deux principes de systématisation sémiologiques ont rivalisé : une série abstraite, la série alphabétique, extérieure à la musique et n'en évoquant pas la nature intervallique ni la spatialisation, mais liée à elle conventionnellement, logiquement et pragmatiquement par les repères habituels de ses actes de production quantifiés (monocorde), et porteuse d'une organisation ordinale constante et rigoureuse (c'est la série qui prime, en tant que suite ordonnée) ; une sériation spatiale géométrique par étagement dans le plan, où on pourra représenter de façon saisissante pour l'imagination les concepts et les relations entre ces concepts, qui constituaient la représentation théorique, la théorie de la musique (c'est l'espace qui prime, en tant que milieu de séparation et d'organisation). Ces 
deux principes vont, pour une plus grande efficacité opératoire, s'unir et coopérer dans la portée. La portée est en effet une conquête tardive, qui résulta de l'interférence positive des deux courants: d'une part celui du développement de l'intuition diastématique des neumes et du déploiement spatial rationnel construit par la théorie, dans lesquels la nature du son dépend de sa position dans l'espace du plan ${ }^{45}$; d'autre part, celui de la connaissance discursive rationnelle qui aboutit à la systématisation monocordale alphabétique, précisant des positions sonores rigoureusement ordonnées. ${ }^{46}$ La synthèse de ces deux courants joint de plus un graphisme neumatique (le punctum) à la conception grammaticale (la lettre) et mathématicogéométrique (le point) de l'unité, pour représenter par un point l'unité sonore du son discret dégagé par la théorie (représentation que l'on trouve d'ailleurs bien avant Gui dans certains diagrammes d'intervalles) ; elle réunit donc l'origine gestuelle et linguistique de la notation : échelonnement dans l'espace et son discret isolé sur une linéarité temporelle. Cette synthèse théorico-pratique est dite avoir été réalisée par Gui d'Arezzo (1784a : 50b), qui, cherchant seulement une méthode pratique de chant et raillant Boèce et les théoriciens "philosophes " sut adapter définitivement à la musique liturgique cette philosophie boécienne. Elle se diffusa du XIème siècle au XIIIème siècle, à partir de l'Italie du Nord et du Centre, vers la France du Nord, les Pays-Bas et les régions rhénanes, puis vers l'Angleterre, le Sud de la France et l'Espagne (Smits Van Waesberghe 1951 : 27-28) ; elle resta en vigueur, jusqu'à nos jours, et son élaboration fut avec raison qualifiée de " phase la plus importante de la musique occidentale » (Wagner $1912: 297$ ).

La portée guidonienne se compose de trois éléments graphiques assurant l'indication précise de la hauteur relative du son: une série de lignes parallèles équidistantes superposées verticalement, en nombre variable au début, mais fixé ultérieurement à quatre pour le chant grégorien et cinq pour la polyphonie; des points élémentaires sur les lignes ou dans les interlignes représentant les sons discrets ; les clefs qui fixent les hauteurs de ces lignes ou de ces intervalles par une lettre monocordale, les premières étant $C=u t$ et $F=f a$, correspondant à la note supérieure des demi-tons de l'échelle diatonique. ${ }^{47}$ Elle est construite par tierces (majeure ou mineure selon la clef) et donne une " carte de l'échelle diatonique ", structure fondamentale de la musique occidentale jusqu'au XXème siècle. Ce système de 
signes, qui ressort à la fois de la représentation imitative, des conventions rationnelles et de la conceptualisation scientifique, est en même temps intuitif et abstrait. Intuitif parce que la portée fournit une vision immédiate des intervalles mélodiques et polyphoniques et que, par son analogie graphique avec la hauteur du son, elle situe immédiatement celui-ci dans l'espace sonore qu'elle visualise ; par une sorte de stratification spatiale de la conscience musicale, elle permet la prévision ; ses montées et ses descentes verticales concrètes, déroulées dans le temps spatialisé selon l'axe horizontal, donnent une image assez bonne du mouvement musical, comme nous le percevons avant que nous analysions notre expérience : elle possède ainsi la représentativité et le pouvoir sur l'imagination qui caractérisaient les neumes. Mais la portée est surtout un système graphique abstrait : elle a rompu avec le geste et n'est plus liée ontologiquement au langage chanté ; elle résulte d'une connaissance objective des phénomènes musicaux, et d'une construction notionnelle spécifique élaborée grâce à des concepts numériques et à des méthodes de mesure expérimentales, et représente la rationalisation théorique définitive de la hauteur du son dans ses variations mélodiques et ses associations polyphoniques ; elle donne ainsi une représentation quantitative précise des relations abstraites entre les sons (ou plutôt d'un ensemble particulier de propriétés sonores et de relations sonores, sélectionnés et abstraits de la complexité du monde sonore). Son évidence ratiostructurelle, tant pour la monodie que pour l'organum naissant, facilitait la lecture, et renforçait la mémorisation audiovisuelle ; elle devient donc un instrument indispensable d'exécution et d'enseignement; et, aidé des syllabes de solmisation et de l'aide-mémoire élémentaire de la " main guidonienne ", elle rendit très accessible au Cantor ignorant le maniement automatisé de notions et de principes qui avaient exigé deux siècles d'études et de réflexion du Musicus. L'efficacité et la primauté de la portée qui supplanta tous les systèmes notationnels antérieurs, ne peut s'expliquer que par ses avantages fonctionnels et la nature de ses rapports avec la musique qu'elle note.

\section{2) Fonctionnement des notations médiévales et rapports entre notation et musique}

a) Rôle opératoire et fonctionnement de la notation

Le passage des neumes à la portée apportait des solutions efficaces aux difficultés de la musique liturgique. Difficultés 
mnémotechniques et pédagogiques, car la notation supplée à la mémoire et supprime le temps et l'espace de communication, conservant de façon permanente les traces durables de ce qui avait paru voué à l'éphémère, et permettant leur transmission lointaine par sa facilité de transport; et la notation précise résultant de cette évolution était ainsi destinée à devenir un intermédiaire fiable entre le créateur et l'exécutant. Difficultés théoriques, car cette notation joua un rôle constitutif, d'une part en précisant l'architectonique modale et en fixant la modalité empirique en une théorie achevée ; d'autre part en donnant la possibilité de noter la polyphonie empirique naissante dont elle permit l'édification architectonique et facilita ainsi les progrès. Mais cette notation sur portée s'avèra un instrument combinatoire d'un tel pouvoir de maniement et de contrôle des sons, et d'une telle puissance d'accueil pour une infinité de leurs combinaisons, que, allant bien au-delà des problèmes du moment qui l'avaient fait élaborer, elle détermina le développement de la musique en composition écrite ; elle provoqua ainsi un élargissement considérable du domaine de cet art en permettant de nouvelles stratégies opératoires qui ouvraient un nouveau champ de possibilités musicales. Perception visuelle fournissant par avance une information sur une perception auditive à reproduire ou à imaginer, elle remplit un double rôle de transmission et d'incitation inductive. Elle transmet la musique sensible existante, ou en imagination, par l'intermédiaire de la pensée intelligible, en agissant par une analyse de la musique, analyse dont elle est à la fois le résultat et l'instrument : cette analyse est rapide grâce à sa ressemblance graphique avec la musique qui la rend facilement saisissable et mémorisable ; elle est sûre grâce à la rationalité théorique de ses conventions, qu'un apprentissage rapide permet d'assimiler. Mais elle n'agit pas seulement par ses capacités analytiques, elle agit aussi par ses capacités de synthèse inductive, et possède ainsi une fonction activement heuristique favorisant la création.

Dans l'accomplissement de ce double rôle, un fossé subsiste entre la réalité musicale perceptive et le schéma visuel informatif, perte d'information musicale dûe à une déperdition de la musique réelle sensible dans la notation abstraite intelligible. Ce résidu de l'ordre du vécu, qui résiste à toute réduction d'une expression vivante à un système de signes (mais que les neumes avaient peut être su en partie assumer), est la cause de l'ambiguïté inhérente entre les signes de la notation guidonienne clas- 
sique et ce qu'ils codifient, et marque les limites de l'adéquation de celle-ci à la musique. ${ }^{48}$ La déperdition du concret musical sensible est répartie sur deux niveaux : une perte au niveau de la symbolisation écrite, dûe à l'imperfection inévitable d'un graphisme dont « les symboles visuels ne donnent au mieux qu'une version schématisée de l'original richement nuancé », et "prix " dont il nous faut "payer " les inestimables avantages de l'écriture " (Sapir 1921: 227) ; mais, l'appauvrissement du signe s'ajoutant à l'appauvrissement du concept, une perte de signification résulte, au niveau de la représentation théorique intermédiaire, de l'approximation et de l'incomplétude d'adéquation de celle-ci par rapport à la musique : la théorie musicale ne donne en effet qu'une représentation abstraite approchée d'une réalité riche et vivante dont elle laisse échapper une partie de la densité existentielle, en mettant en avant, aux dépens des autres caractères, le caractère pertinent de hauteur.

La notation guidonienne classique, j'y insiste de nouveau, représente avant tout la théorie qui a permis son élaboration : la note ne renvoie pas au son concret, comme on le croit souvent, ${ }^{49}$ mais au son conceptualisé, et la note n'est signe du son que si musique et sons concrets restent dans les cadres de la théorie qui sous-tend la notation; le système de signes de la notation correspond au système de sons que décrit la théorie. En conséquence, ce système notationnel se charge de tous les défauts et de toutes les déficiences de la théorie, de même qu'il est la courroie de transmission de sa valeur opérationnelle et inductive et de son efficacité technique. La validité de la notation, en même temps qu'elle est liée à une certaine validité intuitive dépendant des relations de significations sémiologiques justifiables sur le plan de la perception, est le résultat de la validité scientifique de la théorie. Et c'est en tant que représentation symbolique de la théorie que la notation sur portée, durcissant la théorie en un cadre fixe et rigide, réduisit le chant grégorien au diatonisme qui lui fit sans doute perdre une partie de sa souplesse dûe aux petits intervalles utilisés empiriquement. Superstructure qui régit ensuite les structures vivantes, la notation contribua à ancrer les principes qu'elle représente (son discret, hauteur quantifiée, échelle); et, par contrainte culturelle, imposa à la pensée musicale, astreinte d'être notable et connaissant par avance quelle sera sa trace, une forme ponctuelle verticalement spatialisée et scalaire ; elle permit la composition, mais, rabattant la musique dans la structure théorique qui la 
sous-tend, elle détermina en partie ce que veut faire le compositeur pendant de longs siècles. ${ }^{50}$

Le lien ratio-structurel entre la musique et la notation sur portée, lien constitué par la théorie musicale qui motive les caractéristiques graphiques de cette notation, dénie le caractère d'arbitraire qu'on lui a souvent attribué, à la suite de comparaisons superficielles voyant dans le signe musical l'analogue du signe linguistique. Cette accusation d'arbitraire est aussi repoussée grâce à la similitude entre ce graphisme et le mouvement musical, similitude héritée des neumes signalée plus haut, qui crée " la liaison qualitative de la musique avec ses signes visibles " et « la rend présente " dans sa notation (Charles 1973 : 7). Cependant, à côté de ceux qui jugent la notation guidonienne traditionnelle " le semblable », " le double silencieux " (Baruzzi $1926: 185$ ) et « la pleine représentation écrite » de la musique (Wiora $1961: 136$ ), nombreux sont ceux qui se refusent à identifier la partition à l'oeuvre musicale et n'admettent pas que la notation protège l'identité de la musique. ${ }^{51}$ Mais en raison de sa technicité même, la notation possède aussi des règles de structure qui lui appartiennent en propre, et elle a donné naissance à des notions qui ne sont que de simples artifices solfégiques (ainsi la notion scalaire d'Hexacorde, notion tardive faisant du procédé pratique de solmisation de Gui d'Arezzo une échelle de six sons qui lui est improprement attribuée). Bien plus, elle est capable de s'émanciper de la musique sensible et de fonctionner de façon autonome : le symbole peut alors usurper le rôle principal et primer la réalité de la musique dans une combinatoire formelle abstraite, détachée de l'expérience vécue et ne renvoyant pas à un effet audible ; à la limite, il s'agit de combinaisons graphiques sans références sonores (Papiermusik) qui conduisent la musique à une facticité de tous temps condamnée. ${ }^{52}$

La notation guidonienne, qui deviendra, en suivant le développement de la théorie, la notation musicale classique, consiste en symboles stables (portée, points, clefs, puis notes figurées, armatures et altérations, signes de mesure etc.) dont les combinaisons sont définies par des règles stables dans des systèmes théoriques intégrés. Elle fonctionne pour le compositeur dans le sens son (imaginé) - symbole, et pour l'exécutant dans le sens symbole - son : d'où l'interaction mutuelle constante entre notation et musique. Si son fonctionnement compositionnel est difficilement analysable, son fonctionnement dans son rôle d'in- 
dication d'exécution peut être décomposé en quatre phases : captage du symbole graphique par l'oeil, traitement intellectuel de l'information donnée d'après un savoir rationnel préalable, envoi de l'ordre de mouvement de production sonore correspondant, contrôle du résultat par l'oreille. Les symboles de la notation sont donc l'articulation entre les concepts théoriques et l'action pratique, l'intermédiaire graphique entre un acte (rôle opératoire) et une représentation (rôle éidétique). On ne peut pas cependant ramener le problème de la notation musicale à un problème de code, dans lequel à un sens signifié (la hauteur de son) correspondrait un symbole signifiant articulé en trois figures (portée, point, clef) qui pourront ultérieurement se charger d'autres significations (durée, altérations etc.). Les rapports entre la musique et sa notation sont, nous le verrons, trop complexes pour être exprimés par le rapport code-message, émetteur-récepteur. ${ }^{53}$

Dans un précédent travail (Duchez 1980 : 181-82), je distinguais dans la notation deux fonctions également opératoires (correspondant en gros aux deux formes, instrumentale et descriptive, de la connaissance) ${ }^{54}$ : l'indication d'action, transmission d'un schéma d'acte pour l'exécution, et l'expression structurelle, représentation descriptive de relations structurelles objectives permettant la composition. Cette distinction, souvent exprimée d'ailleurs sous des formes différentes qui la recoupent sans la recouvrir exactement, ${ }^{55}$ correspond aux deux modes de fonctionnement du symbole musical notationnel qui, en reprenant les termes de la linguistique, peut agir comme signal et/ou comme signe : comme signal opérationnel, il transmet l'information pratique nécessaire à l'exécution, il déclenche une action qui produit la musique sans en exprimer le contenu ni la structure ; comme signe expressif, il est lié au concept théorique et porteur de signification musicale, il tend à exprimer les structures de la musique qu'il représente. Le signe est généralement aussi un signal, mais le signal peut fonctionner sans être signe expressif (par exemple les signes de tablature). Signal et signe sont réunis dans les symboles (en trois éléments) de la notation guidonienne ; et dans ce système graphique, les deux fonctions opératoires de la notation sont entrelacées, ce qui ne peut pas nous étonner puisque nous avons vu que, selon Piaget, ${ }^{56}$ la seconde résulte de l'intériorisation logico-mathématique de la première, mais ce qui explique son extraordinaire réussite.

La notation classique sur portée agit en effet comme com- 
munication indicatrice d'action, assurant ainsi l'exécution, qui peut être différée dans l'espace et le temps, et fournissant la mémorisation pédagogique qui avait été sa première raison d'être. Mais cette mémorisation était analytique et combinatoire et la notation sur portée a vite dépassé son rôle d'auxiliaire de la mémoire du chant liturgique pour devenir essentielle à la composition musicale qu'elle rendait possible; ${ }^{57}$ ceci grâce à son pouvoir d'expression structurelle, dû à son origine théorique, qui lui permet d'agir comme représentation substitutrice. ${ }^{58}$ Tendant vers une précision structurelle rigoureuse, la notation sur portée tend à " resituer la présence du contenu (musical) signifié " (Derrida $1972: 43$ ) dans une représentation approchée de l'univers sonore vécu; et cette presque-présence de la musique au signe musical lui permet de représenter mentalement et de fixer progressivement les images sonores de l'intention musicale, développant ainsi puissamment les possibilités de la pensée créative et autorisant son organisation en composition complexe. Trace par laquelle on agit et sur laquelle on peut agir, aucune ouvre musicale des dix siècles de musique occidentale qui nous ont précédés n'aurait pu voir le jour sans la notation guidonienne. Nécessaire à l'exécution en tant qu'indication d'action, elle est indispensable à la création en tant qu'expression ; ces deux fonctions entre lesquelles elle oscille, ne peuvent y être séparées et leur réunion intriquée est, plutôt que le simple rapport code-message, la caractéristique de la notation occidentale et la raison de sa fécondité.

Cette étude fonctionnelle de la notation permet de préciser maintenant la nature des rapports entre la musique et la notation, rapports qui, à partir des neumes et en passant par les principaux essais notationnels des IXème-XIème siècles, exigèrent l'élaboration et imposèrent l'usage de la notation sur portée.

b) Rapports entre la notation et la musique des neumes à la portée

J'ai dit dans ce qui précède que chaque notation établit avec la musique qu'elle note des rapports particuliers, selon les relations théoriques qu'elle cherche à exprimer: il n'y a pas de rapport général son-signe pour les notations médiévales des IXème-XIème siècles, puisque dans leurs conditions d'élaboration ce rapport dépend, j'espère l'avoir montré clairement, de la représentation intermédiaire qui évolue radicalement des 
neumes à la portée. Et ces conditions spécifiques d'élaboration d'une notation rationnelle pour une musique vocale dont "l'instrument " de production et de contrôle était le système organique naturel voix-oreille, ont été la cause des rapports spécifiques entre la notation classique et la musique occidentale jusqu'au XXème siècle, malgré l'évolution de l'une et de l'autre.

La différence des rapports notation-musique pour les notations des neumes à la portée - qui toutes, faut-il le rappeler, ne peuvent et ne cherchent à noter que des valeurs relatives de la hauteur du son (et il en sera de même ultérieurement quand on voudra noter les valeurs de durée) — est déjà manifeste dans leur système terminologique, exprimant le rapport son-signe. Pour les neumes, le signe graphique possède un nom qui lui est lié par une ressemblance hiéroglyphique, mais le son musical n'étant pas objectivé, il n'y a évidemment pas de nom de son correspondant ; pour la notation dasienne, le signe est désigné par sa description (attitude semblable à celle des neumateurs), mais le son théorisé correspondant est désigné par le nom du ton liturgique dont il est le finale (Protus ou Archoos, deuterus, tritus, tetrardus), ou par un signe de la notation alphabétique; pour celle-ci le signe alphabétique se nomme lui-même, le nom du son théorique est le plus souvent le nom fonctionnel correspondant dans l'échelle grecque (Proslambanomenos, Hypate des Hypates, etc.) ; et dans la notation guidonienne hautement théorisée, les noms du signe et du son sont confondus dans la syllabe de solmisation, qui leur a été liée conventionnellement par un artifice mnémonique. La différence des rapports notationmusique correspond à la différence des facultés psychiques auxquelles ces notations font appel pour leur réception par l'interprète ; du passage de la tradition orale à la composition écrite, la mémoire est peu à peu libérée de son lourd fardeau de transmission par des méthodes d'appréhension de la musique de plus en plus logiques et rationnelles, mettant en jeu les capacités de synthèse visuelle : tandis que les neumes faisaient surtout appel à la mémoire, ainsi qu'à l'imagination audio-visuelle, pour remémorer le rythme général de la phrase musicale, les notations dasienne et alphabétique qui permettent de solfier un chant non entendu en en indiquant les intervalles exacts, font appel, plus qu'à la mémoire, à un savoir préliminaire ; le savoir exigé est plus étendu, cohérent et plus rationalisé pour la notation sur portée, notation mixte permettant d'innover, qui exige peu de mémoire mais une imagination audio-visuelle développée. L'im- 
portance de l'imagination audio-visuelle, assurant rapidement la connexion - selon un souvenir (pour les neumes) ou selon un savoir préalablement assimilé (pour la portée) - d'une perception visuelle, saisie d'un seul coup d'oil par le lecteur, et d'une perception auditive, réponse orale demandée par le notateur, laisse une certaine indétermination au rapport de transmission notation-musique : celui-ci est en partie une relation humaine notateur (créateur)-interprète (exécutant), relation complexe qui ne peut être réduite à la relation code-message. Cette relation aúdio-visuelle entre le notateur et l'interprète, différente pour les différentes notations des IXème-XIème siècles, porte la modification de l'écoute qui résulte du changement théorique de la conception des sons, des neumes à la portée.

Le rapport de transmission et de communication notateurinterprète indiquant l'exécution, est intrinsèquement lié au rapport d'expression et de représentation, instrument de création, puisque ces deux fonctions sont associées, et il en est la conséquence. Le rapport d'expression et de représentation entre la notation et la musique, rapport que j'appellerai rapport représentatif, détermine donc l'efficacité opératoire de la notation, sa valeur notationnelle. Il est le résult at du fonctionnement de la notation; mais, tandis que l'étude du fonctionnement montre comment la notation transmet la musique, l'étude du rapport représentatif, exprimé par les conditions de fonctionnement (en tenant compte de l'approximation consciente de la notation), et par les résultats du fonctionnement (en tenant compte de l'adéquation limitée de la notation), montre pourquoi cette notation peut transmettre la musique. Ce rapport se modifie des neumes à la portée dans sa forme de saisie, dans ses éléments, dans ses résultats. Son évolution est complexe car le rapport entre la notation et la musique n'est pas, nous l'avons vu, un simple rapport signifiant-signifié ${ }^{59}$ : la notation n'étant pas la représentation graphique directe de la musique, mais la représentation de sa représentation conceptuelle, le rapport représentatif de la notation à la réalité musicale qu'elle connote est le résultat de la combinatoire de deux rapports de nature différente :

1. le rapport épistémologique du système de représentation conceptuelle à la musique qu'il représente ;

2. le rapport sémiologique du système de signes de la notation au système de représentation conceptuelle qu'il dénote.

Le rapport épistémologique entre la représentation concep- 
tuelle et la musique est d'abord intuitif et qualitatif à la période préthéorique des neumes, liée à la représentation grammaticale de la musique ; il devient de plus en plus rationnel, abstrait et quantitatif alors que se développe la pensée théorique. De ce rapport dépend la rationalité et l'adéquation de la notation : plus la musique est rationalisée et plus sa théorie est adaptée avec précision à la musique, plus la notation est précise et adéquate. Et ce rapport n'a été rationnellement déterminé que lorsque la théorie de la hauteur a été complètement élaborée, c'est-à-dire au XIème siècle : d'où la fixation de la notation au XIème siècle.

Le rapport sémiologique entre la notation et la représentation conceptuelle de la musique dépend de la nature et de la fonction des signes de notation. Ce rapport est pour les neumes une correspondance parallèle entre la représentation intuitive et le graphisme qui agit directement sur l'imagination. Il est une convention abstraite pour la notation dasienne qui représente une théorie tétracordale quantitative mais insuffisamment rationalisée et inadaptable aux modes grégoriens. Il est, pour la notation alphabétique, une correspondance logique avec une représentation conceptuelle quantitative rationnelle (échelle monocordale pythagoricienne), adaptable aux modes grégoriens. Pour la notation guidonienne sur portée qui représente le stade définitif de la rationalisation spatio-verticale quantitative et scalaire de la musique, le rapport sémiologique est un compromis entre le parallèlisme concret du graphisme et de la représentation intuitive qui caractérise les neumes, et la correspondance logique rationnelle de la notation alphabétique.

Le rapport représentatif entre la notation et la musique résultant des deux précédents, est pour les neumes, caractérisés par une certaine analogie graphisme-mélodie, une relation de ressemblance non rationalisée, impliquant un contact immédiat signe-son (par mimétisme intuitif) et une écriture bien visualisée mais peu précise. Arbitraire et peu viable pour la notation dasienne, non ressemblante à la musique et mal visualisée, dont la rationalisation tétracordale était erronée, le rapport représentatif est pour la notation alphabétique, sans ressemblance intuitive avec la musique, un rapport purement rationnel et rigoureusement quantitatif, résultant d'un fonctionnement analytique déterminé avec exactitude, mais que son abstraction rendait peu apte à la pratique musicale courante. Pour la notation sur portée, le rapport représentatif précis et constant réunit, dans la représentation de la hauteur des sons, l'analogie imita- 
tive concrète et la rationalité analytique quantitative, en une image graphique structurellement ressemblante; et il réalise, dans une évidence psychologique qui la rend assimilable et manipulable, leur synthèse audio-visuelle. De la validité et de l'efficience de ce rapport représentatif notation-musique, la notation classique tire sa prégnance psychique, son efficacité technique et le pouvoir de réception et d'évolution qui ont assuré sa perdurabilité dans notre culture.

Le passage des neumes à la portée s'effectua dans la foulée d'un changement radical de la sensibilité et de la pensée musicales. Cette transformation fondamentale de l'attitude auditive et du comportement musical consiste, en résumé, dans la substitution au monde de la représentation graphique directe de la perception sensible qualitative de la musique (neumes), d'une représentation graphique quantitative précise, mais aussi qualitativement évocatrice de la perception auditive (notation spatioverticale sur portée), par l'intermédiaire d'une représentation conceptuelle abstraite des éléments quantifiables de la musique (théorie monocordale intervallique et scalaire de la hauteur du son discret). Pour cette transformation, le savoir hérité des Grecs a en quelque sorte "soufflé " aux médiévaux ce qu'ils devaient entendre dans leur chants pour pouvoir les écrire ; mais la pensée médiévale a réussi là où la pensée grecque avait échoué, en réalisant la liaison rationnelle et audio-visuelle de la théorie mathématique de la musique et de sa sémiologie graphique évocatrice.

L'évolution graphique des neumes à la portée ne pouvait pas apporter que des bienfaits : par la rupture de l'inflexion neumatique en sons discrets et par l'immobilisation du mouvement de la voix en repères sans durée dans le cadre de la portée, le musicien médiéval perdait, par le moyen même de la conservation, ce qu'il avait voulu conserver : l'élan de la pensée vocale grégorienne qui "progresse et dure ". Mais cette perte dans la création d'une forme musicale ouvrait à la musique une ère nouvelle, non seulement en lui donnant les moyens de sa diffusion, mais en permettant le développement extraordinaire de son contenu. Le système guidonien acquit une universalité et un rayonnement qui ont persisté pendant presque un millénaire. Il s'adapta si bien et transmit si efficacement la théorie et la musique occidentales qu'elles ont pu s'imposer partout; et la notation sur portée, se chargeant des modifications et des additions nécessaires, a subsisté jusqu'à l'épuisement de cette théorie et de cette musique. 


\section{NOTES}

1. Cet article est le texte d'une communication présentée à la table ronde de paléographie, organisée par le C.N.R.S. (Centre National de la Recherche Scientifique) français, à Orléans les 6 et 7 septembre 1982 dont les Actes publieront le résumé.

2. Comme l'ont bien montré les travaux de psychologie linguistique et religieuse de Marcel Jousse (1925).

3. Saint-Augustin : "Sonus autem, quia sensibilis res est praeterfluit in praeteritum imprimiturque memoriae, rationabili mendacio jam poetis favent ratione, Jovis et Memoriae filias Musas esse confictum est. Unde ista disciplina sensus intellectusque particeps « musicae » nomen invenit " (1948 : L. II, XVI, 41, 434-35).

4. Isidore de Séville : « . . et dicta musica per derivationem a Musis ... sonus quia sensibilis res est et praeterfluit in praeteritum tempus imprimiturque memoriae. Inde a poetis Jovis et Memoriae filias Musas esse confictum est. Nisi enim ab homine memoria tenantur soni pereunt, quia scribi non possunt " (1911: L. III, 15, 1).

5. Aurélien de Réomé : « eoquod haec ars, nisi memoria infigatur, non retineatur" (1975:61).

6. Notons que la mémoire " empirique » en général était extrêmement développée au Moyen-Âge : ainsi Ordorannus de Sens expose de mémoire (repetita memoria), dit-il, le grand monocorde de Boèce qui comprend un très grand nombre de termes grecs et de chiffres. Cf. Ordorannus de Sens 1972: 202-03.

7. Expression de Laloum $1973: 24$.

8. Selon Gui d'Arezzo : "Nam si illi pro suis apud Deum devotissime intercedunt magistris, qui hactenus ab eis vix decennio cantandi imperfectum scientiam consequi potuerunt ..." (1784a: 43b).

9. Cf. les Regulae de arte musica attribuées autrefois à Gui de Cherlieu (1864: 150 a), mais qui sont sans doute de Gui d'Eu ou le Longpont (d'après Cocheril $1959: 372$ ).

10. J'ai ébauché l'étude de ces procédés de descriptions verbales et de leurs sources in Duchez 1981.

11. Pour un exposé plus détaillé de cette conception grammaticale et de la description accentuelle de la musique, cf. Duchez 1981.

12. Cf. manuscrit Rome Bibl. Vat., Palat, lat. 235, fol. 38v (Xe s.) : "... de accentibus toni oritur nota quae dicitur neuma", cité par Wagner $1912: 355$.

13. Sur cette élaboration, cf. Duchez $1981: 572-77$ et tableau II, ainsi que Duchez 1979 : 64-67 pour une autre approche succincte du problème.

14. Depuis Dom A. Mocquereau qui voit dans les neumes une " notation chironomique " (1889), on a longtemps cru que la notation neumatique s'était développée à partir de la chironomie (O. Fleischer, $P$. 
Wagner, E. Jammers, etc.). Helmut Hucke a récemment réfuté cette hypothèse ainsi que l'expression " neumes chironomiques » si souvent employée. Cf. Hucke 1979 : 1-16.

15. Selon la théorie de Piaget (1979:58). J'aurais à revenir sur les théories de la psychogenèse des connaissances de Piaget qui apporte des lumières précieuses sur les processus de la connaissance de la musique et sur l'élaboration de sa théorie et de sa notation.

16. Aurélien de Réomé 1975 : chap. 19, 118-28. Dans le chapitre 19 de ce texte, la description des tons liturgiques est à la fois accentuelle (accentus, acutus, gravis circumflexus) et motrice (inflexio, connexio, producere, erigere, de primere, elevare, deponere, ictum facete ou peragere, etc.). Handschin (1950:69-91) a interprété ce texte en termes neumatiques et suggère une notation paléo-franque, origine de la neumatique aquitaine.

17. Saint-Augustin : "Sed quia verberato aere statim transuent, nec diutius manent quam sonant, instituta sunt per litteras signa verborum. Ita voces oculis ostenduntur non per seipsas, sed per signa quaedam sua. " (1949 : L. II, IV, 5, 242-43).

18. Gui d'Arezzo : "At si littera vel color neumis non intererit, tale erit quasi funem dum non habet puteus " (1784b:31).

19. Il est difficile d'apprécier leur valeur de mémorisation sans une connaissance précise de la mémoire musicale (déclenchement, fonctionnement, objets privilégiés, résultats) dont les neumes sont le recours (étude en cours aujourd'hui).

20. Signalons que le IXème siècle qui eut recours à la théorie pour parer à l'oubli de la musique, créa le mythe de la mémoire musicale : Jean Scot Erigène, répété par Rémy d'Auxerre, suivi par Réginon de Prüm et par le traité anonyme de la Musica Enchiriadis, interpréta en effet dans ce sens le mythe d'Orphée : la perte d'Eurydice, qui représente les principes rationnels de la musique (ars musica in suis profundissimis rationalibus) et Orphée, la puissance de la musique sensible (pulchra vox, optima vox), ne peut arracher à l'oubli des Enfers (inmemor). Cf. Jean Scot Erigène (1939 : 192-93, glose 480, 19), Rémy d'Auxerre (1965: II, 310) ; Réginon de Prüm (1784: 264a, 1962: 57). Ce mythe de la mémoire musicale est traité en détail dans ma thèse de doctorat d'Etat en cours : Imago mundi, naissance de la théorie musicale occidentale dans les commentaires carolingiens de Martianus Capella.

21. L'ethnomusicologie confirme cette relation entre la notation de la musique et sa description par le discours, scientifique ou non. Lorsque Charles Seeger, cherchant à élaborer un procédé de notation qui décrive les musiques non-européennes, reproche à notre notation traditionnelle d'en être incapable, son reproche est vain car il ne tient pas compte du fait que cette notation implique déjà, par son origine, un processus descriptif, celui de la théorie musicale occidentale, et ne peut donc fournir une description différente. Cf. Seeger 1952:360-70 et 1958 : 104-25. 
22. Les traités ne donnent pas de véritable théorie du rythme, organisant et permettant de noter les durées et le tempo qui, du XVème siècle au début du XVIIème siècle, évoluent du tactus à la bat tue et de la notation proportionnelle à la mesure classique ; ils donnent plutôt une suite de systèmes conceptuels ayant chacun leur organisation propre, et articulés par la permanence des problèmes et la continuité des traditions.

23. Je traite en détail cette question dans ma thèse Imago mundi ...

24. Sur cette nécessité pratique de décrire avant de noter, avant de transcrire, nécessité constatée dans les tentatives de notation de toute musique de tradition orale, cf. Rouget $1970: 677-705$.

25. J'ai étudié succinctement ce problème in Duchez $1980: 177-82$.

26. Cf. Franco Donatoni dans son introduction à l'exposition " Ecritures musicales" présentée à la Sorbonne (Paris) en septembredécembre 1979 (p. 30).

27. J'ai utilisé, après les classiques de la linguistique (Saussure, Morris, Marrinet, Sapir) : Buyssens, Jakobson, Mounin, Prieto, etc. .

28. Notons cependant une différence essentielle: les signes de l'écriture linguistique sont de nature phonétique, tandis que les signes de l'écriture musicale résultent de mesures numériques expérimentales.

29. Expression concernant les signes linguistiques et convenant exactement au signe musical et à la combinatoire de la notation. Cf. Mounin $1970: 70$.

30. J'ai étudié sommairement la formation conceptuelle de cet espace théorique de représentation in Duchez 1979.

31. Les premières allusions à une écriture musicale théorique sont exposées dans ma thèse Imago mundi ...

32. En gros, au début du IXème siècle, l'outillage mental du cantor se limitait aux tonaires et aux formules modales ; et les connaissances spéculatives courantes mais non actualisées du musicus, se limitaient à la théorie pythagoricienne des consonances, aux échelles musicales grecques et au diatonisme platonicien.

33. J'utilise encore ici les travaux d'épistémologie génétique de Piaget (cf. note 15) dont j'expose les possibilités d'utilisation féconde pour l'étude des aspects cognitifs de la musique et de la pensée musicale, dans l'ouvrage en voie d'achèvement : Fondements pour une épistémologie historique de la pensée théorico-musicale.

34. L'union de la qualité et de la quantité dans le fait musical est déjà affirmée au milieu du IXème siècle par Jean Scot Erigène : «Ut enim organicum melos ex diversis vocum qualitatibus et quantitatibus conficitur » (1853:637d) ; elle est explicitée lorsqu'est achevée la mathématisation de la mélodie grégorienne au début du XIème siècle : "Quodcirca musicus motus continet qualitatem et quantitatem. Quod si desierit qualitatem quantitatemque motus habere, jam non musicus motus erit. Qualitas autem motus est, utrum si protus vel deuterus aut quilibet alios motus. Quantitas autem utrumnam sit duplus, sesquialter aut 
sesquitertius. Igitur qualqitas in modorum speciebus, quantitas in magnitudine prescribitur motuum Preterea et modorum diversae species non minima prepollent quantiate seu qualitate " (Traité anonyme attribué à Gui d'Arezzo, in Gerbert 1784 : II, 39b).

35. J'analyse longement l'influence de la cosmologie arithmomusicale néoplatonicienne transmise de l'Antiquité sur la formation de la théorie musicale occidentale dans ma thèse Imago mundi . . .

36. Sur l'élaboration du concept de hauteur, cf. Duchez 1979.

37. On trouve déjà cette notion chez Jean Scot Erigène dans ses Annotationes in Marcianum (1939 : 208, glose 501.9).

38. Cf. les travaux de J. Smits Van Waesberghe sur la notation alphabétique dans les traités théoriques, et ceux de Solange Corbin et de Don Hesbert sur les manuscrits grégoriens contenant cette notation.

39. Avec dans les cas les plus nombreux comme note la plus grave $\mathrm{A}=\mathrm{La}$.

40. Avec en général comme note la plus grave $\mathrm{C}=\mathrm{Do}$.

41. Cf. Boèce 1867 : L. IV, chap. 6-12, 318-35.

42. Cf. Boèce 1867 : chap. 17, 345-48.

43. C'est l'opinion de Riemann in $1878 \mathrm{a}: 42,1878 \mathrm{~b}: 609-13$ et 80610 , et $1909: 4$.

44. Cf. Pseudo-Odon (fin Xème-début XIème) : "Litteras monochordi sicut per eas cantilena discurrit ante oculus pone » (1784:255a).

45. Cf. Hucbald : «Qualitatem sive positionem quarumcumque vocum ... (178,4:104a).

46. Cf. Gui d'Arezzo : "Ita igitur disponuntur voces, ut unusquisque sonus, quantumlibet in cantu repetatur ; in uno semper et suo ordine inveniatur. Quos ordines ut melius possis descernere, spissae ducuntur lineae, et quidam ordines vocum in ipsis fiunt lineis, quidam vero inter lineas in medio intervallo et spatio linearum. Quanticumque ergo soni in una linea vel in uno sunt spatio, omnes similiter sonant. Ut autem et illud intelligas, quantae lineae vel spatia unum habent sonum, quantae lineae vel spatia unum habent sonum quibusdam lineis vel spatiis quaedam litterae de monochordo praefiguntur ...» (1975: 66-67).

47. Les sons Do et Fa étaient aussi indiqués dans la théorie de Gui par des lignes colorées ( $\mathrm{C}$ jaune et $\mathrm{Fa}$ rouge) dont l'usage disparut au XIIème siècle.

48. Ajoutons que ce résidu différencie la notation musicale classique, bien qu'élaborée rationnellement et soumise à un système de règles sémantiques et syntaxiques, des symboliques artificielles des sciences expérimentales, auxquelles elle s'apparente par sa conception théorique et sa combinatoire abstraite, mais qui, elles, ne tolèrent aucune déperdition de signification.

49. Ainsi Nattiez (1973: 7-8) qui semble court-circuiter le passage son-note et sauter le " détour théorique ». 
50. Cette coercition de la notation a été souvent dénoncée par les créateurs contemporains. Citons par exemple Stockhausen : "La composition a dû s'en tenir toujours plus à ce qui peut être fixé graphiquement : la représentation sonore toujours plus réduite à ce qu'un système de signes optiques peut transmettre ". (1973:95). Mais d'autres comme Boulez jugent équitablement que la notation, "fondée sur des critères rationnels " est un code qui donne " une base »et qui « est suffisamment flexible pour supporter une charge irrationnelle émotionnelle et affective qui peut le transformer complètement » (1975:95).

51. Cf. Schaeffer $1966: 350$, Chailley $1967: 8$, et, plus récemment, Clifton, critiquant l'adéquation pièce musicale-partition affirmée par Nelson Goodman (Language of Arts), in $1968: 83$.

52. Ce risque de facticité dans la combinaison des signes abstraits a toujours excité la méfiance envers la notation musicale depuis Aristoxène de Tarente (1902: 130-32) jusqu'à François-Bernard Mâche (1963 : 583) en passant par J.-J. Rousseau (1768, article Note et 1753 : 290). Cependant l'autonomie de la graphie musicale " prenant presque d'elle-même sa propre voie " a été considérée comme une conquête positive par certains compositeurs contemporains composant des partitions de lecture, ainsi Stockhausen 1973 : 102-04.

53. La conception de la notation musicale comme un code est extrêmement répandue actuellement : du côté des théoriciens, citons Flechter (1957: 76-96) qui expose cette conception à laquelle s'oppose Cazden (1961 : 117) ; du côté des créateurs, Boulez voit dans la notation un code " chargé d'éléments qui non pas le détruisent mais en tout cas le modifient et lui donnent des inflexions multiples" (1975:83).

54. Cf. la distinction épistémologique opérative-figurative de Piaget (1967: 30-33) ou la distinction linguistique performativeconstatative de Austin (1971: 188-204).

55. Ainsi l'opposition partition d'exécution (représentant les opérations) - partition d'intention (fondée sur les structures) de Schaeffer (1966 : 491-92) ; l'opposition prescriptive notation (comment se faire entendre) - descriptive notation (comment s'entend) de Seeger (1958) ; l'oppostion executive notation-conceptual notation de Cazden (1961 : 114-17) ; l'opposition performance guide - structural guide de Goodman (1968) ; l'opposition Vorschrift (prescription d'acte à exécuter, écriture d'action) - Vorstellung (représentation) et Entwurfschrift (écriture de projet, d'intention) de Stockhausen (1973: 96).

56. Cf. supra notes 15 et 33 .

57. Rappelons que le terme composition, étranger au monde grégorien, ne s'est jamais appliqué à la monodie, mais, jusqu'au XVIIIème siècle, Rameau compris, à la polyphonie et au contrepoint : c'est l'acte de composer les voix, les parties.

58. J'emprunte la distinction husserlienne entre indice (Anzeichen) ou communication indicatrice, et expression (Ausdruck) ou représenta- 
tion substitutive à l'analyse pénétrante de la double fonction du signe par Derrida (1972 : chap. 1, 18-27 et 105).

59. Les paragraphes suivants développent et complètent des arguments présentés rapidement in Duchez 1980 : 182.

\section{RÉFÉRENCES}

\section{SOURCES ANTIQUES ET MÉDIÉVALES}

\section{ANONYME}

1965 : Alia musica (Traité de musique du IX ${ }^{e}$ siècle), éd. J. Chailley. Paris : Institut de musicologie de l'Université de Paris.

1981: Musica et scolica Enchiriadis una cum aliquibus tractatulis adjunctis, éd. H. Schmid. Veröffentlichungen der Musikhistorischen Kommission, Vol. III. Munich : Bayerische Akademie der Wissenschaften.

\section{ARISTOXÈNE DE TARENTE}

1902: The Harmonics of Aristoxenus, éd. et trad. H.S. Macran. Oxford : Oxford University Press.

\section{AURÉLIEN DE RÉOME}

1975 : Musica Disciplina, éd. L. Gushee. Corpus Scriptorum Musica BOÈCE 21. Rome : American Institute of Musicology.

1867 : De institutione arithmetica libro duo. De institutione musica libri quinque, éd., G. Friedlein. Leipzig : Teubner; rep. 1966.

\section{CASSIODORE}

1937 : Cassiodori senatoris institutiones, éd. R.A.B. Mynors. Oxford : Clarendon Press.

\section{GERBERT, M.}

1784: Scriptores ecclesiastici de musica, 3 vols. Typis St. Blasien;

\section{GUI D'AREZZO} rep. 1963.

1784a : Epistolia Michaeli monacho de ignoto cantu, in Gerbert 1784, II, 43-50.

1784b: Regulae musicae rhythmicae, in Gerbert 1784, II, 25-34.

1955 : Micrologus, éd. J. Smits Van Waesberghe. Corpus Scriptorum de Musica 4. Rome : American Institute of Musicology.

1975 : Tres tractatuli Guidonis Aretini. Guidonis Prologus in antiphonarium, éd. J. Smits Van Waesberghe. Divitiae Musicae Artis, A. III. Buren : Frits Knuf. 
GUI DE CHERLIEU (Pseudo)

1864: Regulae de arte musica, in É. De Coussemaker, Scriptorum de Musica medii aevi nova series, II, 150-92. Paris : Durand; rep. 1963.

HERMANN CONTRACT

1784 : Opuscula musica, in Gerbert 1784, II, 124-53.

HUCBALD DE SAINT AMAND

1784: De harmonica institutione, in Gerbert 1784, I, 103-52.

ISIDORE DE SEVILLE

1911: Isidori hispalensis episcopi Etymoloharium sive originum libri XX, 2 vols., éd. E.M. Lindsay. Oxford : Clarendon Press.

JEAN SCOT ERIGÈNE

1853 : De divisione naturae, éd. J.-P. Migne. Patrologiae latinae, Vol. CXXII, 430-1022.

1939 : Annotationes in Marcianum, éd. C. Lutz. Cambridge : The Medieval Academy of America.

ODON DE CLUNY (Pseudo)

1784: Dialogus de musica, in Gerbert 1784, I, 251-64. ODORANNUS DE SENS

1972 : Opera omnia, éd. R.H. Beautier. Paris : Institut de Recherche et d'Histoire des textes (CNRS) ; Chap. VI, « De divisione monochordi ", édité, traduit et commenté par M.-E. Duchez, 202-25.

RHABAN MAUR

s.d. : De reum naturis et verborum proprietatibus et de mystica rerum significatione ou De universo, éd. J.-P. Migne. Patrologiae latinae, Vol. CXI, 1-614; rep. 1976.

RÉGINON DE PRÜM

1784: De harmonica institutione, in Gerbert 1784, I, 230-47. RÉMI D'AUXERRE

1965 : Commentum in Martianum Capella, 2 vols., éd. C.E. Lutz. Leiden : E.J. Brill.

SAINT AUGUSTIN

1948 : De ordine, éd. et trad. fr. R. Jolivet. Bibliothèque augustinienne 4. Paris : Desclée de Brouwer.

1949 : De doctrina christiana, éd. et trad. fr. G. Combes et J. Farges. Bibliothèque augustinienne 11. Paris : Desclée de Brouwer.

\section{RÉFÉRENCES MODERNES}

AUSTIN, J.L.

1971: The Philosophy of Language. Londres : Macmillan. BACHELARD, G.

1928 : Essai sur la connaissance approchée. Paris : Vrin.

1949 : Le rationalisme appliqué. Paris : Presses Universitaires de France. 
BARUZZI, J.

1926 : "Pensée musicale et graphisme ", Journal de Psychologie normale et Pathologique, XXIII, 185-197.

BOULEZ, P.

1975 : Par volonté et par hasard. Paris : Seuil.

CARDINE, E. (Dom)

1957 : «Théoriciens et théoriciens : À propos de quelques exemples d'élision dans la mélodie grégorienne ", Études Grégoriennes, II, 27-35.

1970 : Conférence à l'office de Radiodiffusion et Télévision Française du 23 mars 1970.

s.d. : Sémiologie grégorienne. Abbaye Saint Pierre de Solesmes.

CAZDEN, N.

1961 : "Staff Notation as a Non-Musical Communication Code", Journal of Music Theory, V/1, 113-28.

CHAILLEY, J.

1967 : La musique et le signe. Lausanne : Édition Rencontres.

CHARLES, D.

1973 : "La musique et l'écriture ", Musique en jeu, $\mathrm{N}^{\circ} 13,3-13$. CLIFTON, T.

1975: "Some Comparisons Between Intuitive and Scientific Descriptions of Music », Journal of Music Theory, XIX/1, 66-111.

COCHERIL, M.

1959 : "Gui de Cherlieu 》, in Encyclopédie Fasquelle de la musique. Paris : Fasquelle, I, 372.

COLE, $\mathrm{H}$.

1974: Sounds and Signs : Aspects of Musical Notation. Oxford : Oxford University Press.

CORBIN, S.

1977: Die Neumen, Vol. I/3. Cologne : Arno Volk-Verlag. Hans Gerig KG.

DADELSEN, G. von

1964 : "Über das Wechselspiel von Musik und Notation", in Gedenkschrift Walter Geistenberg. Kassel : Bärenreiter, 17-25.

DERRIDA, J.

1972 : La voix et le phénomène. Paris : Presses Universitaires de France.

DUCHEZ, M.-E.

1979 : "La représentation spatio-verticale du caractère musical grave-aïgu et l'élaboration de la notion de hauteur de son dans la conscience musicale occidentale ", Acta Musicologica, LI/1, 54-73. 
1980 : "La représentation de la musique : Information d'action et expression structurelle dans la représentation de la musique occidentale traditionnelle ", in Actes du XVIII Congrès des Sociétés de Philosophie de langue française, Strasbourg, Juillet 1980, éd. Centre National de Recherche Scientifique, résumé : 177-82.

1981: "Description grammaticale et description arithmétique des phénomènes musicaux : le tournant du $\mathrm{IX}^{\mathrm{e}}$ siècle ", in Sprache und Erkenntnis im Mittelalter, Miscellanea Medievali (Veröffentlichungen des Thomas-Instituts des Universität zu Köln), XIII/2, 561-79.

FLECHTER, S.

1957 : "Music Reading Reconsidered as a Code-Learning », Journal of Music Theory, I/1, 76-96.

GOODMAN, N.

1968 : Language of Arts : An Approach to a Theory of Symbol. Indianapolis : Bobbs Merril.

HANDSCHIN, J.

1950: "Eine alte Neumenschrift », Acta Musicologica, XXII/1, 69-97.

HUCKE, $\mathrm{H}$.

1979 : "Die Cheironomie und die Entstehung der Neumenschrift", Die Musikforschung, XXXII/1, 1-16.

JOUSSE, M.

1925: Le style oral rhythmique et mnémotechnique chez les Verbo-moteurs. Paris : Archives de Philosophie III, cahier 4.

KUNZE, S.

1974 : "Raumvorstellung in der Musik », Archiv für Musikwissenschaft, XXXI/1, 1-21.

LALOUM, C.

1973 : « À bâtons rompus : Ethnomusicologie et musiques graphiques ", Musique en jeu, $\mathrm{N}^{\circ} 13,24-35$.

MÂCHE, F.-B.

1963 : " Le son et la musique ", Mercure de France, CCCXLIX/1201, 582-98.

MOCQUEREAU, A. (Dom)

1889: Paléographie musicale, I, Solesmes.

MOUNIN, G.

1970: Introduction à la sémiologie. Paris : Éditions de Minuit. NATTIEZ, J.-J.

1973 : "Sémiologie et sémiographie musicale », Musique en jeu, $\mathrm{N}^{\mathrm{o}} 13,78-85$.

PIAGET, J.

1967 : "L'épistémologie et ses variétés ", in Logique et connaissance scientifique, Enclyclopédie de la Pléiade. Paris : Gallimard, 3-61. 
1979 : Théorie du langage, Théorie de l'apprentissage, Débat entre Jean Piaget et Noam Chomsky, organisé par Massimo Piattelli-Palmarini, Centre de Royaumont pour une science RIEMANN, $\mathrm{H}$. de l'Homme, Octobre 1975. Paris : Seuil.

1878a : Studien zur Geschichte der Notenschrift. Leipzig : Breitkopf und Härtel; rep. 1970.

1878b : "Die Uranfange der deutschen Tabulatur », Allgemeine musikalischen Zeitung, XXXIX, 609-13 et 806-10.

1909 : Die byzantinischen Notenschrift im 10. bis 15. Jahrhundert. Leipzig : Breitkopf und Härtel.

ROUGET, G.

1970 : "Transcrire ou décrire », in Pouillon, J. et Maranda, P., éds., Échange et Communications (Mélanges offerts à Claude Lévi-Strauss). Paris-La Haye : Mouton, Vol. I : 677-705.

ROUSSEAU, J.-J.

1753 : Lettre sur la musique française. Paris; rep. in Écrits sur la musique. Paris : Stock-Musique, 1979, 251-323.

1768 : "Notes », in Dictionnaire de Musique. Paris : Duchesne. SAPIR, E.

1921: "The Musical Foundations of Verse », Journal of English and Germanic Philology, XX/1, 213-28.

SCHAEFFER, $P$.

1966 : Traité des objets musicaux. Paris : Seuil.

SEEGER, C.

1952 : "Preface to the Description of Music », in Kongressbericht der Internationalen Gesellschaft für Musikwissenschaft. Amsterdam : Alsbach and Co., 360-70.

1958 : "Prescriptive and Descriptive Music-Writing ", The Musical Quarterly, XLIV/2, 184-95.

SMITS VAN WAESBERGHE, J.

1951: "The Musical Notation of Guido of Arezzo », Musica Disciplina, V, 15-63.

STOCKHAUSEN, K.

1973 : "Musique et graphisme (1959) ", Musique en jeu, $\mathrm{N}^{\circ} 13$, 94-104.

WAGNER, $\mathrm{P}$.

1912: Einführung in die gregorianischen Melodien, II : Neumenkunde Palaographie des liturgischen Gesanges, 2nd ed. Leipzig-Breitkopf und Härtel; rep. 1962.

WIORA, W.

1961: Les quatre âges de la musique, trad. par J. GaudefroyDemembynes. Paris : Payot. 